Aus der Universitätsklinik für Ohren-, Nasen- und Kehlkopfkrankheiten (Direktor: Geh. Med.-Rat Prof. Denker) und dem pharmakologischen Institut der Universität in Halle a. S. (Direktor: Prof. Dr. Kochmann).

\title{
Kritischer Beitrag zur Frage der Wirksamkeit des Vuzinum bihydrochloricum bei endolumbaler Anwendung als Heil- mittel gegen Meningitis.
}

Von Dr. med. H. Birkholz, ehem. Assistenzarzt der Klinik.

Die Behandlung der eitrigen Hirnhautentzündungen, die von infizierten Organkomplexen an der Schädelbasis, besonders vom Felsenbein her, entweder direkt oder unter $Z$ wischenschaltung anderer intrakranieller Prozesse, induziert werden, ist bekanntlich im Gegensatz zu ihrer Diagnostik, die besonders durch grundsätzlich frühzeitige und dann evtl. systematisch kontrollierende Anwendung der in den Händen des Erfahrenen als harmloser Eingriff zu bezeichnenden Lumbalpunktion und durch genaueste Untersuchung und Würdigung des Lumbalflüssigkeitszustandes zu großer Sicherheit gediehen ist, noch immer ein unbefriedigendes Gebiet.

Das chirurgische Prinzip, den primären Herd auszuschalten, hat durch die Kriegserfahrungen an Schädelschüssen mit Freilegung des Endokraniums eine Bestätigung seiner Berechtigung im großen erfahren; hier handelte es sich um traumatische Infektionen vorzugsweise der Konvexität des Hirns (95b). Bei der òperativen Behandlung : derselben erwiesen sich die Aussichten auf Erfolg von einer Reihe von Faktoren abhängig, die zu beeinflussen nicht ausschließlich in der Hand des Operateurs liegen. Der Umfang der durch das Trauma gesetzten Gewebsveränderungen in der Fläche und Tiefe, die Schwere der Infelktion, die individuelle Abwehrkraft des Organismus sind ganz allgemein die Schnelligkeit der Ausbreitung einer Entzündung bedingende Kräfte, gegen welche selbst ein frühzeitiges, breites Freilegen und Ausräumen des primären Wundherdes ein nutzloses Vorgehen sein kann. Dagegen ist es als feststehend zu erachten, daß bei Neigung zur Abgrenzung des Infektes der rechtzeitigen Herdfreilegung der Wert 
eines die Heilung fördernden Momentes von hervorragender Bedeutung zukommt. Da im Einzelfall die exogenen und endogenen Faktoren der Infektion sich nicht im voraus bestimmen lassen, ist in der Praxis das chirurgische Verfahren bei perforierenden Traumen der Schädelkapsel mit Recht als wichtigste vorbeugend-antimeningitische Behandlungsart anerkannt (I6).

Betrachtet man von diesem Gesichtspunkt aus die Chancen der chirurgischen Behandlung der otogenen Meningitis, so wird man, wie groß auch hinsichtlich der Lokalisation, der Art der Entstehung und des pathologischen und klinischen Verlaufes bei diesen Infektionen der basalen Hirnhäute die Unterschiede zur traumatischen Kriegsmeningitis im einzelnen sein mögen und müssen, in manchem, und zwar besonders hinsichtlich der Prognosenstellung, eine gewisse Verwandtschaft feststellen. Erinnern wir uns an den Jansenschen (53) Grundsatz, die ersten meningealen Symptome als letzte Warnung und Mahnung zur Operation des erkrankten Ohres anzusehen, der $u$. a. von Körner (6r), Heine (40), unterstrichen wurde und von Denker $(26 \mathrm{~b})$ dahin präzisiert wird: ,Es ist dringend indiziert, bei dem ersten Auftreten meningitischer Symptome sofort den Herd im Mittelohr durch die Aufmeißelung und bei chronischer Mittelohreiterung durch die Radikaloperation, bei Labyrintheiterung durch breite Eröffnung des Labyrinths auszuschalten!" Hiermit ist dasselbe Prinzip der Frühoperation ausgesprochen, das für die traumatische Meningitis maßgebend ist.

$\mathrm{DaB}$ die Frühoperation bei dieser Indikationsstellung in unserem Gebiete günstige Aussichten haben muß, leuchtet ein, wenn man bedenkt, daß in den bei weitem zahlreichsten, meningitische Zeichen darbietenden Fällen noch keine eigentliche Meningitis bestehen dürfte. Wir wissen heute, daß meningeale Reizzustände, welche so im Vordergrunde stehen und klinisch so schwer sein können, daß sie von beginnender Meningitis klinisch kaum unterscheidbar sind, die aber dennoch mit einer anatomisch nachweisbaren, ausgebreiteten Erkrankung der. Hirnhäute nichts zu tun haben, bei durchaus außerhalb der Dura liegenden entzündlichen Prozessen vorkommen; z. B. bei perisinuösen Abszessen, Sinusphlebitis und -Thrombose, ja unter Umständen schon bei heftiger Mastoiditis - z. B. kleiner Kinder - - vorkommen können. Andererseits lehrt die Erfahrung, da $B$ auch subdurale und intraarachnoidale Phlegmonen und enzephalitische Prozesse (bisweilen auch Encephalitis lethargica) unter Umständen klinische Zeichen von Meningitis darbieten können, ohne daß eine nennenswerte Beteiligung der weichen Hirnhäute tatsächlich statthätte. Anatomisch handelt es sich dabei teils um ein einfaches Volumen liquoris auctum, eine rein irritative Funktionsstörung, teils um lokalisierte „Minimal- 
Meningitis" (Borries II6), teils um umschriebene Wegleitungen und abgekapselte Herde; was diesen anatomisch-physiologischen Vorgängen das klinische Bild der beginnenden oder ausgebildeten Meningitis verleiht, ist lediglich die begleitende, kollaterale (,,sympathische"), an sich völlig harmlose (pressorische?) Reizung der Meningen in der näheren Umgebung des Herdes (Ödem des Sehnervenkopfes, Kulenkampfsches Zeichen bzw. Bruczinskische Reflexkontraktur, Nackenstarre) und deren spinale Ausläufer (Kernig, Hyperästhesie im Gebiet der unteren Extremitäten). Dies wird jeweils durch den Befund einer lediglich unter vermehrtem Druck stehenden, zytologisch und chemisch kaum veränderten Lumbalflüssigkeit erhärtet. Diese Zustände mit Körner (6I) als ,Vorstufen“ echter Meningitis anzusprechen, geht nicht ohne weiteres an; es dürfte sich in allen solchen Fällen um eine mehr minder akute Arachnoidalaffektion mit ursächlichem Vorherrschen des Intoxikationsanteiles handeln; dafür spricht auch die Tatsache, daß umschriebene Herde in den Maschen der Spinnwebenhaut sich öfter bei Vorliegen chronischer Otitiden, deren Erreger bzw. Unterhalter man als in ihrer Virulenz abgeschwächte, wenn nicht gar saprophytäre Elemente (z. B. Proteus) ansehen muß, als bei akuten Mittelohraffektionen entwickeln (Denker 26a); ebenso haben die Erreger bei meningealwärts vordringenden Hirnabszessen, wofern solche'nicht gar steril befunden werden, in der Regel als wenig virulent zu gelten. Die hieraus resultierende mangelnde Tendenz zur Generalisierung - und nur eine ausgebreitete Hirnhautentzündung verdient den alten, von der makroskopischen pathologischen Anatomie eingeführten Namen „Meningitis“ - wird in solchen Fällen durch die rechtzeitige Operation unterstützt.

Fassen wir den Meningitisbegriff aber in dieser ursprünglichen Form und fügen wir ihm als seinem wichtigsten modernen Kriterium in vivo den Gehalt des Lumbalpunktates an polymorfkernigen Eiterzellen und Bakterien von sicherer Virulenz hinzu, so können wir es uns erklären, da $B$ bei solchen Infektionen die chirurgische Therapie auch bei frïhzeitiger Anwendung viel geringere Chancen haben muß als in den oben genannten Fällen von Nichtmeningitis. Wie in der Literatur der Kriegschirurgie, so existieren auch in der unsrigen tatsächlich nur wenige Fälle derartiger Meningitiden, die allein durch chirurgische Inangriffnahme des primären Herdes der Heilung zugeführt wurden. Das schnelle Uberschreiten der von der Pachymeninx gebildeten Grenze sowie eines etwa innerhalb der weichen Hirnhäute durch Ödem, Leukozyten und Fibrinverklebungen gebildeten Schutzwalles läßt sich eben allermeist bei foudroyant verlaufenden Infektionen, besonders labyrinthogener, weniger ossaler- Herkunft, ebensowenig als bei schwerer Kriegsmeningitis schnell genug durch Herdausschaltung verhindern. Wenn man es sich dennoch zur Regel macht, zu operieren, 
wird man sich immer der geringen prognostischen Aussicht des Eingriffes bewußt bleiben müssen.

Es ist daher nur zu erklärlich, daß in der Otorhinologie die Verhütung der Meningitis, sei es durch prophylaktische chirurgische Eingriffe, wie sie z. B. bei mit einer Mittelohreiterung komplizierten Fraktur der Schädelbasis bzw. des Petrosum von mancher Seite (36, I04a) empfohlen werden und bei perforierenden Verletzungen des Felsenbeines Grundsatz sind (69, 104c), sei es medikamentös(Urotropin), im allgemeinen eine größere Rolle spielt, als die mehrir minder aussichtslos erscheinenden Versuche der therapeutischen Beeinflussung einer bereits manifesten Infektion der Hirnhäute. Daran aber, daß aus der - anscheinend - gelungenen Verhütung ein bindender Schluß auf die Wirksamkeit eines therapeutischen Faktors, wie auf den übrigen medizinischen Gebieten, so auch in dem hier in Rede stehenden gar nicht, oder doch nur auf Grund ganz besonders umfangreicher, objektiv gesammelter Erfahrungen gezogen werden kann, dürfte festzuhalten sein. Dieser Grundsatz wird heutzutage bei dem Massenansturm neuer pharmazeutischer Präparate von allen ernsten Kritikern betont; ich erinnere an die lichtvollen Ausführungen von $\mathrm{His}$ (44), gelegentlich des Streites um das Friedmannsche Tuberkuloseheilmittel am Io. II. 20 in einer Aussprachevers. in Berlin betr. allg. Heilmittelkritik. Von unserer Seite hat übrigens schon Hinsberg I9I2 (43) beim Kapitel der Urotropin-Wirksamkeit bei Meningitis auf das Unzulässige eines Schlusses aus verhütender Wirkung hingewiesen. Gerade bei der Beurteilung therapeutischer Wirkung auf die Meningen müssen wir um so vorsichtiger sein, als wir ihnen ebenso wie anderen serösen Häuten (Peritoneum) eine erhebliche Widerstandskraft gegen bakterielle Invasion zusprechen müssen, kraft derer sie auch noch ziemlich ausgedehnte entzündliche Prozesse zur Heilung zu bringen vermögen (Streit, 95).

Wesentlich anders liegen die Dinge naturgemä $\beta$ bei meningitischen Infektionen, die rein in das Gebiet des Internisten gehören. Bekanntlich ist der Infektionsweg bei diesen vorzugsweise ein hämatogener. So gut als bewiesen dürfte dies für die septischen und für die Pneumokokken-Meningitiden sein, die sich mitunter an genuine Lungenentzündung anschließen. Die Herkunft und der Infektionsweg der kontagiösen Meningitis, sei es nun, daß sie in epi- oder endemischer Form auftritt oder sporadisch vorkommt, sei es, daß sie vom Meningokokkus intrazellularis in seiner grampositiven oder -negativen Varietät oder von anderen Erregern (65) hervorgerufen wird, ist dagegen noch eine wissenschaftliche Streitfrage. Allen internistischen Meningitiden ist zum mindesten ein wichtiger Punkt gemeinsam, der, daß sie nicht mit einer Herdausschaltung chirurgisch angreifbar sind; ob die Invasion 
durch Sanierung der Eingangspforten (sept. Herd, Lunge) und des Blutes auf medikamentöse Weise zu unterbrechen gelingt, ist eine andere, hier zunächst nicht interessierende Frage.

Es ist jedenfalls kein Wunder, daß wir der inneren Medizin in der Hauptsache die Erfindung und den Ausbau derjenigen Behandlungsweisen verdanken, die die erkrankten Hirnhäute direkt anzugreifen suchen, ich meine, die Anwendung der Lumbalpunktion und der Hirnpunktion $\mathrm{zu}$ therapeutischen Zwecken, Methoden, die neuerdings in Form systematischer subarachnoidaler Behandlung eifrig gepflegt werden. Ein kurzer Uberblick über sie sei hier gestattet.

Die endolumbale Therapie verfolgt bei den entzündlichen Erkrankungen der weichen Hirnhäute vornehmlich drei leitende Ideen:

I. das rein mechanische Prinzip, Entzündungsprodukte, Giftstoffe exogener und endogener Herkunft, evtl. geformte Erreger zu entfernen, vorübergehend die Hirnsubstanz vom Exsudatdruck zu entlasten, sie selbst wie die Hirnhäute zu erhöhter Abwehr zu befähigen, die Selbsterneuerung des Liquor zu beschleunigen und eine der Heilung günstige Hyperämie zu erzeugen. Hierzu dienen folgende Verfahren:

a) die gehäufte ,einfache“, evtl. ,große“ therapeutische Lumbalpunktion, d. i. I-2 mal tägliches Ablassen von Liquor, und zwar- nicht nur bis roo mm Liquordruck - welche Zahl meist als Norm hingestellt wird - , sondern nach Bedarf bis zum Erreichen negativer Druckwerte. Von Quincke (85) mit großem Optimismus zunächst bei der tuberkulösen Meningitis angewendet, wurde sie von Lenhartz (64) in die innere Medizin eingeführt. Heutzutage ist, wie dauernde ermutigende Mitteilungen in der Literatur beweisen $(29,55,74,39)$, ihre Domäne vor allem die Meningokokken-Meningitis, in deren moderner. Therapie sie obenan steht und, obzwar besonders in Verbindung mit anderen endolumbalen Behandlungsweisen, befriedigende Erfolge erzielt. Die in der Literatur zusammengestellten Fälle von Heilungen tuberkulöser Meningitiden durch Lumbalpunktion beschränken sich auf einige zwanzig ( $74,9 \mathrm{a})$, doch wird von allen Autoren, des ominösen Ausganges ungeachtet, auffällige subjektive Besserung angegeben, die allein schon zur Rechtfertigung der (symptomatischen) Therapie zu dienen hat. Die durch die gewöhnlichen Eitererreger, weniger die durch Pneumokokken verursachten eitrigen Meningitiden, deren Prognose gar als infauster denn die der tuberkulösen gilt (50), werden kaum beeinflußt. Die Erfolge von Rolly (87) dürften ganz vereinzelt dastehen.

Seitens der Chirurgen empfiehlt, so weit ich sehe, zuletzt Klapp (57) bei traumatischer spinaler Meningitis (laminektomierte Rückenmarksschüsse) große Lumbalpunktionen, und zwar nach dem Vorgange Biers. (II), der die hyperämisierende Wirkung dadurch zu erhöhen trachtete, mit gleichzeitiger Kopfstauung. Im übrigen hört man von. 
dieser Kombination, die früher schon von Vorschütz (103) bei epidemischer Meningitis mit angeblich gutem Erfolge angewendet wurde, sehr wenig.

Dem Beispiele Babinskis, der (46) als erster zielbewußt die therapeutische Lumbalpunktion bei Ohrenkrankheiten angewandt hat, folgten alsbald andere Autoren. Bönninghaus (I4) hält sie bei Meningitis serosa solange für leistungsfähig, als ein äußerer Hydrozephalus mit oder ohne inneren besteht. Als einer der ersten(Ig) und überzeugtesten Anhänger hat Brieger zu gelten, der (I9a, Schlußwort) in der Lumbalpunktion ,das wichtigste Moment . . . . für die Therapie der otogenen Meningitis " erblickt. In der Folgezeit wird ihr Wert durchweg ziemlich hoch angeschlagen. Nur sehr wenige Autoren verhalten sich ablehnend $(75 \mathrm{a}, 47)$, andere zeigen gewisse Resignation $(6 \mathrm{I}, 54,79)$, die Mehrzahl, und gerade die neueren Autoren (40, IoI a, 6, 24, I04b, 30, I0, 95) stimmen auf Grund von günstigen eigenen Erfahrungen in einer Reihe von Fällen, die zu kritisieren mir fern liegt, etwa in dem Urteil von Denker überein, der in seinem Lehrbuche der Lumbalpunktion, selbstverständlich nächst der Ausräumung des induzierenden Herdes im Felsenbein, die erste Stelle bei der Meningitisbekämpfung einräumt. Aus der neuesten Literatur erwähne ich, daß Bondy (I5a) über Heilung streptogener (3 Fälle), Goording (34) pneumogener (I Fall) und Döderlein (27) (I Fall) staphylogener Meningitis durch mehrfache Lumbalpunktion berichtet. Fleischmann (30) und Soyka (93) empfehlen sie mit neuer theoretischer Begründung. So interessant übrigens die von Fleischmann gegebene Zusammenstellung otogener Meningitisfälle, in denen die Lumbalpunktion als alleiniger Heilfaktor in Frage kommt, ist, so verliert sie doch bei näherem Zusehen erheblich an Wert, dánn nämlich, wenn man den Bakteriengehalt der Lumbalflüssigkeit als das sicherste und eigentlich einzig maßgebend sein sollende Kriterium heranzieht. Nun finde ich aber, daß einige der von ihm angeführten Fälle in der Originalliteratur als steril angegeben sind (Alt I, Knapp 58), andere (Schulze 92) die Möglichkeit einer akzidentellen Bakterienverunreinigung zulassen, wieder andere (Bondy I5, Gradenigo 35) von den Autoren als wenig virulent angesprochen wurden. An der generell sehr schlechten Prognose der bakteriellen ausgebreiteten Meningitiden dürfte auch heute ebensowenig zu zweifeln sein, wie es seinerzeit Körner, Haymann, Preysing, Mygind (6I, 4I, 84, 75a) statistisch feststellten; besonders gilt das von der Streptokokken-, dann von der Staphylokokken-, endlich von der Pneumokokken-Infektion.

b) Die Ventrikelpunktion. Bei kindlichem Hydrozephalus schon früher ausgeführt, wurde sie von Bergmann (8) zuerst in einem Fall von tuberkulöser Meningitis, obzwar erfolglos, angewendet; nach 
Bönnighaus (1. c.) ist der Eingriff durch den intakten Schädel hindurch bis I897 häufiger, doch im ganzen kaum öfter als 25 mal (38), vorgenommen worden, davon $4 \mathrm{mal}$ mit Erfolg bei Meningitis serosa (I4, Kasuistik), einmal von Bönninghaus selbst. Neißer-Pollack (76) haben das Verfahren, die Seitenventrikel vom Schädeldach bzw. von der Schläfe her aus nach Trepanation zu punktieren, nicht nur zu diagnostischen Zwecken angewendet. Ihr Schüler Westenhöffer (I06) hält sie auf Grund großer Erfahrungen bei epidemischer Meningitis dann für unbedingt angezeigt, wenn ein starker Hydrops ventriculorum anzunehmen ist. Seitdem ist es sehr stille über die Ventrikelpunktion geworden. Chirurgische Werke (9) erwähnen sie nur bei der Behandlung des infantilen Hydrozephalus.

In der Behandlung otogener Meningitiden spielt sie den Lehrbüchern zufolge (40, S. 227) keine bedeutende Rolle mehr. Erst neuerdings scheint sie wieder mehr in Betracht gezogen zu werden. So erkennt Fleischmann (30) unter besonderer Würdigung ihrer Gefahren ihr gewisse Vorteile vor der Lumbalpunktion zu, dann nämlich, wenn ausgesprochener innerer meningitischer Hydrozephalus vorliegt. Die Begründung seiner Empfehlung stützt sich indessen nur auf theoretische Erwägungen. Viel aktiver und optimistischer ist Soyka (93), da er aus Erfahrungen in zwei einschlägigen Fällen den Schluß zieht, bei Punktio lumbalis sicca sei sofort die Ventrikelpunktion vorzunehmen. Der Literatur zufolge wird seitens der Internisten heute die Ventrikelpunktion nur noch selten angewandt. Lewkowicz (70) u. a. versuchen sie neuerdings in Verbindung mit intrakameralen Seruminjektionen bei epidemischer Meningitis.

DaB permastoidale Punktionen des Seitenhorns der Hirnkammer oder sogar des vierten Ventrikels gelegentlich und mehr minder zufällig bei Operationen otogener intrakranieller Komplikationen eine therapeutische Indikation durch Liquorablaß erfüllt haben mögen, sei nebenher erwähnt.

c) die lumbale Drainage der Cysterna terminalis, zunächst von Quincke (85), als subkutane, dann (Lit. bei Fleischmann) als äußere Liquorableitung vorgenommen, sowie die therapeutische Laminektomie (Friedrich, Göbel, 26b) dürften bei Meningitis heute zu den seltensten Maßnahmen gehören, da die Technik dieser Verfahren kompliziert, dieInfektionsgefahr groß ist und die Erfolge. nicht ermutigend wirken.

d) die Ventrikeldrainage nach außen ist (30) otologischerseits bisher nur einmal, von Dench, bei Hirnhautentzündung verwendet worden; hier handelte es sich anscheinend um eine seröse Meningitis, es ist daher nicht auffallend, da $B$ der Fall geheilt wurde; auch der Nutzen dieses Verfahrens steht keinesfalls im Verhältnis zu seiner 
Gefährlichkeit. Die innere Drainage, die nach dem Prinzip von Payr, Anton, v. Bramannu. a. Ventrikelhöhlensystem und Subarachnoidalraum verbinden soll, ist auffallenderweise für druckentlastende $Z_{w}$ wecke bei Meningitis noch nicht angewendet worden. Ich möchte glauben, daß ihr Wert bei seröser Ventrikel-Meningitis, wenn wir den Ausdruck festhalten wollen, gleich etwa dem der Bönninghausschen Ventrikelpunktion einzuschätzen ist. Bei eitriger Meningitis dürfte sie geradezu kontraindiziert sein.

e) die grundsätzlich kraniale subarachnoidale Drainage nach außen bei schwerer otogener Meningitis war früher verbreiteter als jetzt. Gegenwärtig dürften die meisten Otologen mit uns auf dem Standpunkt stehen, daß bei labyrinthogener Arachnitis die spontane Entleerung von Hirnflüssigkeit, die bei der Neumannschen, besonders aber nach Uffenordescher Labyrinthresektion (IOI, IOI b) teils aus dem Kochleastumpf, teils durch Einreißen der Hirnhäute im, Fundus- des Meatus acusticus internus $\mathrm{zu}$ entstehen pflegt, zur Entlastung ausreicht, in manchen Fällen auch noch durch leichtes Schlitzen der Dura zu verbessern ist; ist ein subduraler AbszeB Ursache der Meningitis, ist die Dura breit zu spalten; obwohl die Neigung des enzephalitisch geschwollenen Hirns, sich aus dem Duraschlitz hervorzudrängen (Denker 26b), den Wert solchen Vorgehens im allgemeinen beeinträchtigt. Liquorabfluß ist also zwar bei labyrinthogener Meningitis als erwünscht hinzunehmen, nicht unbedingt aber bei ossalem Infektionsweg. Einen grundsätzlich aktiveren Standpunkt nimmt bis zur heutigen Zeit die nordische Schule ein ( 7 ; 7I, 90). Durch Holmgren (46) wurde die Drainage der Kleinhirnbrückenwinkel- und der basalen Ponszysterne mittels Mullstreifen nach Spaltung der Dura der hinteren Schädelgrube unter Intaktlassen des Labyrinthes eingeführt und die Indikationsstellung dahin ausgedehnt, daß auch bei nichtotogener, besonders auch epidemischer Meningitis bei erfolgloser Lumbalpunktionsbehandlung auf diesem Wege vorzugehen ist $(3,98)$. In Analogie dazu wird von Murphy; Westenhöffer (I06) und neuerdings Eden (28) eine Drainage der Cysterna cerebello-medullaris bei eitriger internistischer Meningitis nach vorangegangener Schlitzung der Membrana atlanto-occipitalis vorgenommen. Otologischerseits hat. Fleischmann (30) dies Verfahren an drei Fällen der Voßschen Klinik nachgeprüft. Zweimal handelte es sich um schwere von Nasennebenhöhlenempyemen aus fortgeleitete Meningitiden, einmal um labyrinthogene Meningitis; in allen drei Fällen wurden außer der Ausschaltung des primären Herdes mehrfache Lumbalpunktionen vorgenommen, also die Meningitis von drei verschiedenen Seiten her bekämpft. Erfolg: Obwohl die Drainage an sich gut war, kamen die Fälle zum Tode. Die Deduktion Fleischmanns, da $B$ sie Hirndrucksymptome vermeidet oder mildert, wird angesichts 
dieser MiBerfolge illusorisch. Ihre Wirkung auf die Infektion scheint tatsächlich außerordentlich gering zu sein; das steht im Einklang mit den kurz zitierten Ergebnissen älterer Drainageversuche. Mechanisch allein ist pathogenen Mikroorganismen in vivo nicht beizukommen.

f) Auswaschungen des Subarachnoidalraumes mit physiologischer Kochsalzlösung und Locke-Ringerscher Flüssigkeit im Anschluß an die Lumbalpunktion werden, soweit ich unterrichtet bin, seitens der Internisten bei epidemischer Meningitis mit großem Nutzen vorgenommen, es sind Fälle beschrieben, bei denen die Auswaschungen, konsequent bis zu mehr als fünfzig Malen ausgeführt, zum guten Ende führten. Allerdings kommt man mehr und mehr resignativ dazu, die indifferenten Flüssigkeiten durch „Desinfizientien“ zu ersetzen, außerdem die Prinzipien der großen Lumbalpunktion und Serumbehandlung dazuzufügen $(33,82)$.

Kraniolumbale (5) und lumbokraniale Durchspülungen des gesamten subarachnoidalen Systems (I7, Ior, 42, 59, 7, I7a), die bei eröffnetem kranialem Subarachnoidalraum und gleichzeitiger Lumbalpunktion auch am Lebenden technischen Schwierigkeiten nicht begegnen sollen, haben bisher mehr theoretisches Interesse als praktischen Erfolg gehabt. - Knick (59) sah allerdings in zwei von fünf Fällen (Staphylokokken-Diplokokken-Pyocyaneusinfektion) Heilerfolg, ebenso Borries-Berggren. (7, I7a) in einem von vier Fällen. Fleischmann, der sie wegen der Gefahr der Keimverschleppung, die er in einem durch kraniolumb. Spülung behandelten Falle beobachtete, "für bedenklich hält, weist mit Recht auf die von Knick aufgestellte Indikation hin, nur in desolaten Fällen mit Bakteriengehalt sei man sie zu versuchem berechtigt; hinzusetzen möchte ich: aber nur dann, wenn man dessen sicher ist, daß die subarachnoidalen Räume miteinander kommunizieren, und dann nur lumbokranialwärts. Mir scheint sogar, daß Versuche in dieser Richtung mit neuen Desinfizientien gemacht. werden sollten (s. u.).

2. Das Prinzip direkter, passiver Immunisierung des Subarachnoidalraumes.

Bei der auf eine jede endolumbale Behandlungsart leichter und erfolgreicher reagierenden Meningitis epidemica gehört die Serumanwendung heutzutage zu den bestbekannten Verfahren. Levis (67) Statistik vom Jahre Igo8 ergibt, bei Auswahl geeigneter Fälle (!), eine Reduktion der Mortalität von $78-100 \%$ auf $10 \%$. Da indessen die Serumbehandlung stets mit großer Lumbalpunktion verbunden ist, neuere Autoren außerdem grundsätzlich den Lumbalsack auswaschen bzw. desinfizieren, läßt sich bei Anlegen einer strengen Kritik über ihren Wert nur so viel sagen, da $B$ auch die Immunotherapie bei alleiniger Anwendung 
nicht vollauf befriedigt. Bei der durch die gewöhnlichen Eitererreger hervorgerufenen eitrigen Meningitis vollends sind der Literatur zufolge die Erfolge einer spezifischen Serumbehandlung, der Schwere der Infektion entsprechend, lange nicht so günstig. Fleischmann (1. c., S: 346) stellt im ganzen sieben fremde Fälle (Pneumokokken, Streptokokken) zusammen, die durch derartige Immunisierung der Heilung zugeführt worden sein sollen; I2 eigene Fälle, die er selbst direkt mit Antistreptokokkenserum, und zwar schon von den ersteri Krankheitstagen an behandelte, blieben trotz mehrmaliger Seruminjektion (IO bis $25 \mathrm{ccm}$ ) und trotz Aufbietens anderer therapeutischer Maßnahmen (Urotropin, Herdausschaltung) völlig unbeeinflußt, eine traumatische (gramnegative Diplokokken!!) Meningitis heilte, eine andere (Ätiologie ?) wurde vorübergehend günstig beeinflußt. Ob diese beiden Erfolge tatsächlich dem Bestehen einer kranialen „,Gegenöffnung“ zukommen, woran Fleischmann denkt, erscheint mir sehr zweifelhaft (s. u.). Fleischmann hat jedenfalls das Verdienst, die I2 Fälle seiner Vorgänger als das hinzustellen, was sie wohl sind: Zufälligkeiten, Subjektivismen.

3. Das Prinzip, auf chemotherapeutischem Wege lokal eine Entwicklungshemmung bzw. Abtötung der Infektionserreger zu erzielen.

Überschaut man die von Preysing, Eskuchenund Fleischmann gegebenen Zusammenstellungen über die derartigen Bestrebungen, so fühlt man sich erst recht dazu geneigt, Preysing recht zu geben, welcher IgI2. ironisch meinte (84), daß offenbar der Rückenmarkskanal sehr viel vertragen kann. Teils zu Injektionen und Infusionen, teils zu Ausspülungen wurden, jeweils dem Stande der Lehre von den Desinfizientien entsprechend, Karbol und Lysol, Quecksilbersublimat und -Oxyzyanat, Urotropinlösungen, Methylenblau, kolloidale Silberpräparate, angewandt. Zum experimentellen Beweis sicher ist keines dieser Mittel. Heutzutage wundern wir uns nicht mehr darüber, wenn ionisierte Metallsalzlösungen im Organismus keine desinfizierende Wirkung zu entfalten vermögen, da das wirksame Metall durch die Eiweißstoffe unschädlich gemacht wird; ähnlich wissen wir, daß die üblichen organischen Desinfektionsmittel durch die Gegenwart gelösten Eiweißes hochgradig in ihrer Wirkung beeinträchtigt werden. So auch Urotropin im meningitischen Liquor (59a). Heute baut man große Hoffnung auf die ,innere Desinfektion" einerseits mit kolloidalen Metallsalzen (Silber, Gold, Krysolgan!), andererseits mit bestimmten, als in vitro hochwertig erkannten organischen Stoffen. Die theoretisch bei Gonokokken und den morfologisch und biologisch verwandten Meningokokken aussichtsreiche Silbertherapie ist neuerdings von Coglievina (23) und Käding (I Fall) erfolgreich mit dem besonders fein verteilten $2 \%$ igen Dispargen, von Eskuchen (29) mit dem Methylenblausilberpräparat Argochrom 
in $2 \%$ iger Lösung bei epidemischer Meningitis fortgesetzt. Über Anwendung bei pyogener Meningitis scheint Literatur nicht vorzuliegen. Eine neue Ära schien mit der Einführung der Chininabkömmlinge zu beginnen, deren bakteriotrope Wirkung bekanntlich von Morgenroth erkannt wurde. Während Chinosol von Schottmüller (9I) ergebnislos angewendet wurde, ist das auf Streptococcus lanceólatus eingestellte Optochin (Abtötungstiter I: 130000 ) bisher von verschicdenen Autoren in insgesamt I4 Fällen als gegen Pneumokokkenmeningitis ,,spezifisch" wirksam befunden ( $107,72,88,4$, I02), auch bei epidemischer Meningitis soll es sich bewährt haben (3I, 63, 4, 77). Friedemann (3I) berechnet sogar die Heilungsaussichten epidemischer Meningitis bei Optochinanw endung auf $90 \%$ gegen $50 \%$ bei Serumanwendung. Das Eucupin, das in vitro sich als mehr auf Strepto- und Staphylokokken eingestellt erwiesen hat (Abtötungstiter I : 20000-I:40000), verwendeten Valentin (I02) in einem und Fleischmann (30, S. 738) in drei bzw. vier Fällen, bisher allerdings ohne Erfolg. Die Kritik Fleischmanns trägt, wie ich weiter unten ausführen werde, zweifellos den Resorptions- und Verteilungsverhältnissèn des Eucupins nicht genügend Rechnung; er irrt auch, wenn er meint, daß eiweißhaltige Medien und Körperzellen die Chininpräparate in ihrer Wirkung nicht beeinträchtigen (s. u.).

Eine Kritik sämtlicher soeben systematisch angeführter Behandlungsarten, die zum Teil hochkompliziert und äußerst ingeniös erdacht sind, soll hier nicht im einzelnen, wie sie Fleischmann vornimmt, stattfinden. Eine jede derartige Beurteilung ist naturgemäß außerordentlich von der individuellen Veranlagung des Kritikers abhängig; Überlegungen kann man in Richtung auf ein pessimistisches oder optimistisches Urteil anstellen (siehe auch His). Mir genügt es, festgestellt zu haben, daß eine große Anzahl von Methoden zur Bekämpfung der Meningitis angegeben worden ist. Dieser Umstand und die stete Suche nach neuen Heilmitteln spricht dafür laut genug, daß keine derselben annähernd als sicher zu betrachten ist. (Vergleiche auch das vorsichtige Urteil von Bieh1 [Io, S. I67] und Linck [69b].)

Wenn neue Behandlungsarten der Meningitis angegeben werden, von denen man annehmen kann, daß sie zum mindesten keinesfalls zu schaden vermögen, wird es unsere Pflicht sein, sie an möglichst großem Krankenmaterial zu erproben und kritisch ihre Wirksamkeit nach jeder Richtung hin zu prüfen. Dabei wird man sich immer wieder vor Augen halten müssen, daß eine exakte Kritik erstens mal nie durch verhütende Anwendung einer Methode gewonnen werden kann, sodann, daß der einzig wirklich brauchbare Prüfstein der Meningitisbeeinflussung nicht der klinische Verlauf allein, sondern der Liquor cerebrospinalis ist, dessen Elemente, um die Modifikation eines Wortes von Frerichs (96) aus der Nierenpathologie zu gebrauchen als ,Bote 
der Vorgänge in den Meningen" zu gelten haben, vorzüglich aber der bakterienhaltige Liquor.

Daß das subarachnoidale Behandlungsprinzip an sich das einzig richtige und aussichtsreiche bei diffusen Meningitiden sein muß, liegt meines Erachtens auf der Hand. Wir wissen heute, daß es ungeheuer schwer ist, eine Substanz zu finden, die bei extralumbaler, und sei es auch intravenöser, Einverleibung mit einiger Sicherheit an der Stätte durch angereicherte Ausscheidung zur Wirksamkeit kommt, wo wir es wünschen, nämlich an den dünnen Hirnhäuten. Da diese, von lipoidlöslichen Substanzen abgesehen, nicht nur unter physiologischen Bedingungen, sondern selbst in erkranktem Zustande für die gebräuchlichen Arzneimittel nicht permeabel sind, werden sie heute im kolloidchemischen Sinne als eine Art von Grenzmembran aufgefaßt (Io8). Neuerdings hat Kochmann (mündliche Mitteilung) dieses anläßlich von Versuchen, durch intrasinuöse Injektion Preglscher Jodlösung: auf die Meningen zu wirken, am Lebenden für Jodverbindungen bei luetischen Erkrankungen der Hirnhäute nachgewiesen, ältere, gegenteilige Angaben, die in die üblichen Lehrbücher übergegangen sind, berichtigend. Praktisch in Betracht kommt für diese Art einer ortsspezifischen Allgemeinbehandlung nur die alte Urotropinmedikation. Da sie wissenschaftlich einigermaßen begründet (25, Iro), und nach dem Urteil erfahrener Kliniker manchmal wirklich von Nutzen ist $(26 \mathrm{a})$, dürfte sie wohl um so mehr allgemein zum Arzneischatz bei der Meningitisbehandlung gehören, als sie kaum $z u$ schaden vermag. Und doch wird es heute keinem mehr einfallen, bei manifester Meningitis vom Urotropin einen sicheren Heilerfolg zu erwarten und sich auf dasselbe $\mathrm{zu}$ verlassen; um so weniger, wenn neuartige aussichtsreiche Behandlungsmethoden in Konkurrenz stehen. Bemerkt sei übrigens, daß auch die theoretischen Grundlagen der Urotropintherapie heute als schwer erschüttert gelten müssen (io, $59 \mathrm{a}, 22$ ).

Eine neue Extralumbalmethode hat vor einigen Jahren dennoch Flèischmann (30) zu propagieren unternommen. Seit Kafka (56) wissen wir, da $\beta$ bei meningealen Reizungs- und Entzündungszuständen Körperimmunstoffe aus dem Serum in den Liquor hinübertransfundieren, was sie unter normalen Verhältnissen niemals tun. Dies gilt vor allem von den Luesreaginen der Wassermannschen Reaktion; während Luetiker mit intaktem Nervensystem auch bei stärkerer Dosierung des Liquor (Hauptmannsches Auswertungsverfahren) keinen luetischen Ambozeptor im Liquor aufweisen, ist bei allen Formen der Lues des Zentralnervensystems einschließlich der sogenannten Metalues der Wassermann im Liquor mehr minder konstant positiv (8r). Bei akuten Entzündungen der. Hirnhäute auf gleich welcher Grundlage fanden ferner Weil und Kafka (I05) im Liquor auf Hammel- 
blut eingestellte Hämolysine, die sonst nur im normalen Serum vorhanden sind; der Gehalt daran geht dem klinischen Entzündungsverlauf parallel, zeigt also jeweils das Stadium der Meningitis dadurch an, da $\beta$ es den Grad der Permeabilität der Meningen bzw. ihres Kapillarendothels ermessen läßt. Ein Gegenstück zur Hämolysinreaktion, auf deren Bedeutung in unserem Gebiet neuerdings Soyka (93) hinweist, ist die von Braun-Husler (I8) angegebene; ihr positiver Ausfall - es handelt sich um eine Fällungsreaktion mit dreihundertstel Normalsalzsäure - soll den Übertritt von Komplement in den Liquor anzeigen und in erster Linie für akute Meningitiden charakteristisch sein. Gestützt auf diese Tatsachen, überschwemmte Fleischmann (1. c.) in sechs Fällen von eitriger Meningitis die Blutbahn durch intravenöse Gabe von je Ioo ccm Höchster Antistreptokokkenserum; tatsächlich konnte er $\mathrm{I}_{2}-24$ Stunden nach der Injektion im Liquor Mengen von Pferdeeiweißstoffen des verwandten Serums nachweisen, die ihm genügend erschienen, um eine therapeutische Beeinflussung der Meningen wahrscheinlich zu machen. Fleischmanns Erfolge entsprechen jedoch nicht seinen theoretischen Voraussetzungen, denn nur einer von sechs Fällen ging in Heilung über. Es will mir möglich erscheinen, daß die gefundenen Pferdeeiweißstoffe nicht mit den injizierten Antikörpern identisch sind, denn die von Fleischmann angewendete Uhlenhuthsche Methode weist lediglich den artfremden Gruppen-Eiweißkörper nach. Bessere Erfolge will übrigens Gerstmann (32) mit Antistaphylokokkenserum gehabt haben.

Neuartig sind die Versuche, durch intravenöse Injektion der modernen inneren Antiseptika eitrige Hirnhautentzündung zu heilen. Nachdem Kobrak I9I6 (60) eine kombinierte Optochin-UrotropinBehandlung per os empfohlen hatte, verwendete SpieB (94) Trypaflavin in einem Falle mit gutem Erfolg. VoB (IO4C) verspricht sich von der Fortsetzung dieser therapeutischen Versuche Erfolge. Wir vermögen a priori diesen Optimismus der Autoren in bezug auf eine extra-lumbale Therapie ebensowenig wie Linck [25a] $\mathrm{zu}$ teilen; in den ungenügenden Erfolgen der intravenösen Salvarsan-Therapie auf die Lues des Zentralnervensystem, die von einigen Neurologen zugegeben werden, sehen wir eine Bestätigung unserer Zweifel.

Uns erschienen die therapeutischen Versuche von Linck $(69 a, b)$ mehr beachtenswert. Linck, der die endolumbale Behandlung so lange für die beste Anwendungsform hält, als wir das ideale innere organspezifische Desinfiziens noch nicht besitzen, hat bekanntlich im vorigen Jahre das Morgenroth-Klappsche Vuzin angewendet und zur Prüfung empfohlen, eine Substanz, die nach Angabe von Morgenroth-Tugendreich spezifisch gegen den Streptokokkus gerichtet ist (Abtötungstiter in vitro I zu 80000) und deren Wirkung durch Eiweiß- 
stoffe nicht in dem Maße beeinträchtigt werden soll, wie die übrigen bekannten Desinfektionsmittel. Auf Grund seiner Erfahrung kam Linck zu der Dosierung, daß er zu kurativen Zwecken jeweils eine Injektion von Io $\mathrm{ccm}$ einer $2 \%$ igen Vuzin-Kochsalzlösung, das ist $20 \mathrm{mgr}$ pro dosi, vornahm. Unter Zugrundelegung eines Mittelwertes von Ioo $\mathrm{ccm}$ (Gumbrecht, Eskuchen) bezw. $200 \mathrm{ccm}$ (Fleischinann) Liquor im gesamten Subarachnoidalraum berechnet sich hieraus die endolumbale Vuzinkonzentration bei schneller gleichmäßiger Verteilung auf I: 5000 bzw. I : Ioooo; bekanntlich forderte Klapp (57c) für seine Tiefenantisepsis eine Konzentration im Gewebe von I : Iooo bis I: Ioooo. Damit erscheint die Lincksche Anwendungsart vom experimentell-bakteriologischen und klinisch-empirischen Standpunkt aus theoretisch als eine innere umschriebene Desinfektion gut fundiert.

Wie gestalten sich nun die bisherigen Erfolge der Vuzinbehandlung? Linck selbst $(69 b)$ berichtet über Anwendung seiner. Lösung in to Fällen; von diesen sind 4, weil in ihnen die Indikation der Prophylaxe gestellt worden war, zu strenger Kritik des Behandlungswertes nicht brauchbar, wie Linck selbst (S. 237) betont (Nichtmeningitisgruppe). Von den 6 Fällen, in denen ein kurativer Zweck erstrebt wurde, wiesen Nummer 3,4 und 7 ein steriles Lumbalpunktat auf; von diesen wurden $2(3,4)$, wie vorauszusehen, autoptisch als Begleitmeningitiden begrenzter infektiöser Arachnitisherde an der Schädelbasis bestätigt; bemerkenswerterweise zeigte Fall 4, eine labyrinthogene Meningitis, eine trügerische vorübergehende Remission nach Vuzin, welches auf die Dauer den Fortschritt der Infektion nicht zu hindern vermochte; eine Wirkung auf die Einfallspforte scheint hier nicht stattgefunden zu haben. Auf den 3. Fall (Nr. 7) mit ähnlichem Lumbalflüssigkeitsbild, der geheilt wurde, einen Analogieschluß zu ziehen, dürfte erlaubt sein, zumal. Linck ihn selbst als eine postoperative Meningitis serosa auffaßt. Diese 3 Fälle, bei denen das Vuzin unmöglich seine bakterizide Wirkung in den abgekapselten Herden entfalten könnte, vielmehr lediglich die spinalen Ausläufer, ev. gar nur das lumbale Hypopyon (Körner, l. c.) der meningitischen Reizung angegriffen haben kann, schalten ebenfalls aus, wenn wir uns an die Bakterienflora als den Prüfstein eines Desinfiziens halten wollen. Von den 3 letzten Fällen Lincks, die durch Bakterienbefund im spinalen Punktat im Einklang mit dem klinischen Bilde als ausgebreitete Meningitiden anzusprechen sind, kamen Fall I und 8, wenn nicht als kausale, so doch als zeitliche Folge, nach mehrmaliger Vuzinisierung zur Heilung; in dem einen Fall wurden Pneumokokken, in dem anderen Staphylokokken gefunden. Der 3. Fall (Fall 2), der Streptokokken aufwies, kam zum Tode. Hinsichtlich seiner muß man sich meines. Erachtens der Linckschen Epikrise voll und ganz anschlie- 
Ben. Bei Betrachtung der beiden geheilten Fälle $x$ und 8 fällt sofort die verschiedene Dosierung des Vuzins auf. Im Fall I wurden drei Injektionen von je ro ccm einer $0,01-0,03 \%$ igen Vuzin-Kochsalzlösung (Vuzinkonzentration im Liquor also I : I 000 000-I : $300000 \mathrm{mit}$ $1 / 10-3 / 10 \mathrm{mg}$ Vuzin), im Fall 8 dagegen vier Injektionen von je Io $\mathrm{ccm}$ $2 \%$ iger Lösung vorgenommen. So erklärlich es auch ist, daß der Patient des ersten Versuches minimale Dosen erhielt, so verliert der Fall eben dadurch an Wert zur Beurteilung des desinfizierenden Effektes. Die berechnete Vuzinkonzentration dürfte nach den Angaben Morgenroths und seiner Schüler für Pneumokokken, von deren Virulenzgrad wir hier einmal absehen wollen, selbst als hemmende Dosis zu klein sein; als Grenzverdünnung (Hemmungsgrenze) finde ich in der Literatur I : 500000 (Tetragenus, Bylsma [20]) und I: 750000 (Diphtheriebazillen [80]). Diese Zahlen gelten indessen für wässerige Lösung; durch Bylsmas exakte Nachprüfung wissen wir abtr, daß bereits Vuzinkochsalzlösung einen niedrigen Grenztiter hat ( $1: 200000$ für Tetragenus $\left.{ }^{1}\right)$ ), daß Aszitesflüssigkeit und Serum den Tieer noch erheblich herabsetzen, daß aber besonders Blutzellen, für die der Teilungskoeffizient bzw. die Affinität des Vuzins noch größer als für gelöstes Eiweiß ist (20, S. 327), die bakteriotrope Wirkung nahezu aufheben. Berückssichtigen wir, daß im meningitischen Liquor nicht nur $7 \%$ Kochsalz, sondern zum mindesten $0,3 \%$ Eiweiße und eine wechselnde Zahl von suspendierten weißen Blutzellen vorhanden ist, so können wir die Vuzinwirkung im Falle I mit Sicherheit ausschließen. Zweifellos liegt hier einer der Fälle vor, der infolge wenig virulenter Erreger und hoher Abwehrstoffkonzentration im Liquor des Kranken zur Spontanheilung tendierte. Ob man nun den Befund des klaren pneumokokkenhaltigen Liquors als Frühstadium (6gb, Seite 238) ansprechen will, stehe dahin; nach den Remissionen zu urteilen, haben Anfälle von akutem Hydrozephalus stattgefunden, die auch lediglich durch die Lumbalpunktionen gebessert sein können, vom toxischen Einfluß auf die Arachnoidea ganz zu schweigen. Auch aus diesen Gründen vermag ich in diesem Fall keinen Beweis für das propter hoc des Vuzins zu sehen. Bei dieser Art der Betrachtung bleibt nur Fall 8. Dieser erscheint allerdings bei unbefangener Beurteilung durchaus beweiskräftig.

Die Kasuistik der endolumbalen Vuzinbehandlung bereicherte neuerdings Zimmermann (Iroa) um einen Fall, der hinsichtlich einiger maßgebender Faktoren (Subarachnoidaldrainage und Lumbalinfusion, Diplokokken [wann?]) dem Fall I entspricht. Die Epikrise

1) Bac. bezw. Microc. tetragenus (Sarcina t.) kommt nach Quincke (85a) auch als seltener Meningitiserreger in Frage. 
berücksichtigt jedoch $\mathrm{m}$. E. zu sehr rein klinische Zeichen, deren absolute Verläßlichkeit nicht feststeht und $\mathrm{m}$. E. daher durch objektive Kriterien ersetzt werden sollte. Der Wert des Falles zur Entscheidung des propter hoc scheint mir durch meine Ergebnisse einigermaßen erschüttert.

Auch wir haben die Anregung Lincks begrüßt und ihre klinische Nachprüfung an einem größtmöglichen Material unter genauester Kontrolle des Lumbalpunktates in Angriff genommen. Vorweg sei bemerkt, daB wir grundsätzlich zu der endolumbalen Vuzinisierung nach Linck die alte perorale Urotropinmedikation mit $3 \mathrm{~g}$ pro Tag hinzufügten. Naturgemä $B$ keine Optimisten bei der Meningitisbehandlung, haben wir uns nicht für berechtigt gehalten, auf ein Mittel, daß unter Umständen vielleicht doch etwas nützen, auf jeden Fall aber sicher nicht schaden kann, lediglich zur wissenschaftlich exakten Erprobung des neuen Mittels zu verzichten. Unsere Erfahrungen sind natürlich noch nicht zum $\mathrm{Ab}$ schluß gekommen. Meinen Liquorprotokollen entnehme ich, daß wir bisher außer in den beiden Fällen, die ausführlich gebracht werden sollen, noch keine Gelegenheit hatten, bakterielle Meningitiden mit Vuzin anzugehen; oftmals handelte es sich um verhütende Anwendung, in anderen Fällen um Begleitmeningitiden, die trotz Sterilität klinisch und zytologisch oft einen schweren Verlauf nahmen. Dies Material wird später zu sichten sein.

Im folgenden werde ich zu berichten haben über einen Krankheitsfall, der mir Veranlassung gab zu dem Versuch, etwas über die Verteilung der Vuzinlösung im subrarachnoidalen Raum in Erfahrung zu bringen. Linck selbst regt ja zu solchen Versuchen, die er für wichtige Kriterien hält, an. Dieser Fall ist, wie sich zeigen wird, auch in Hinsicht auf andere Fragestellungen von Interesse. Ein zweiter Fall gab mir alsbald die erwünschte Gelegenheit, die begonnenen Studien besonders in der Richtung auf das Problem zu vertiefen: „Kann Vuzin im Lumbalsack überhaupt eine Heilwirkung bei Meningitis durch eine Art von Desinfektion erzielen?" Ein dritter Fall diene als kasuistischer Beitrag.

Uber das Verhalten von endolumbal einverleibten Substanzen am Lebenden sind unsere Kenntnisse im allgemeinen recht lückenhaft. Das liegt in der Hauptsache daran, daß wir über Grundfragen der Liquorphysiologie noch immer nicht sicher orientiert, sondern widerspruchsvollen Lehrmeinungen ausgesetzt sind. Wollen wir ganz ehrlich sein, so müssen wir auf Grund der reichen Literatur, die seit Quincke über dieses Thema vorliegt, sagen, daß wir auch heute nicht mit Sicherheit wissen, woher der Liquor stammt (Plexus? Pialvenen? Hirnsubstanz?), als was er aufzufassen ist (Sekret? Transsudat? Lymphflüssigkeit?), welchen Strömungsweg er einschlägt und wo und wie er zur 
Resorption kommt. Rehm (8I) gibt das „Nescimus" offen in Umrissen zu und rät zum Kompromi $B$. Obwohl mit den verschiedenensten Theorien operiert wird, wissen wir noch nicht einmal mit Sicherheit, ob das Foramen Magendie, Ventrikelsystem und Subarachnoidalraum miteinander verbindend, im Leben besteht! Über die Art der Liquorströmung können wir lediglich in großen Umrissen mit einiger Sicherheit annehmen, daß ein bestimmter Sekretionsdruck einerseits, eine Resorptionstendenz an anderen Orten sie in Gang erhält, daß dieselbe für gewöhnlich sehr langsam (56) und in kraniokaudaler Richtung verläuft. Besonders der letzte Faktor muB bei Anwendung lokaler Therapie mit Hilfe der Lumbalinfusion in Rechnung gestellt werden. Auf Einzelheiten, die insonderheit über die resorptiven Kräfte im Subarachnoidalraum bekannt sind, einzugehen, behalte ich mir im Zusammenhang mit den Ergebnissen meiner Untersuchungen vor; vorläufig sei nur hervorgehoben, daß die Pathologie des Liquor quoad Entstehung, Strömung und Verbleib noch vielmals komplizierter ist, als die an sich nicht absolut klare Lehre von seinen physiologischen Bedingungen.

Der erste Fall, der zu diesen Fragen Stellung. zu nehmen gestattet, ist auszugsweise folgender:

K., Käthe, I2 Jahre, Aufnahme II. IV. 2I.

Kam in die Klinik wegen angeblich sechswöchentlicher Ohreiterung rechts mit Schwindelerscheinungen. Befund: reichlich eitrige, nicht fötide Absonderung rechts aus einem großen Trommelfelldefekt; von vorn und oben kommen reichlich Granulationen aus den epitympanischen Räumen; keine Erscheinungen von seiten des Vestibularapparates.

Am Tage nach der Aufnahme wurden die Granualtionen mit der Schlinge entfernt und zunächst nichts Auffälliges beobachtet. Am Spätnachmittag plötzlich Erbrechen, Temperaturanstieg auf über $39 \mathrm{Grad}$, Spontannystagmus nach links und starker Schwindel. Die sofort vorgenommene genaueste Untersuchung des Vestibularis und Kochlearis ergab mit aller wünschenswerter Deutlichkeit die Zeichen einer akuten Destruktion der im Labyrinth suspendierten Endorgane, wahrscheinlich also Labyrintheinbruch. Da die erhöhte Temperatur auf Beteiligung der Meningen hindeutete, wurde trotz fehlender klinischer Symptome sofort die Lumbalpunktion vorgenommen. Sie ergab unter $300 \mathrm{~mm}$ Wasserüberdruck stehenden makroskopisch klaren globulinaren Liquor. In der Zählkammer waren mit Sicherheit unter 25 weißen Blutzellen im $\mathrm{ccm}$ I8 polymorphkernige Leukozyten. Blutbeimischung war spärlich vorhanden, doch ließ sich die Leukozytenzahl auf keinen Fall daraufhin zurückführen (um jeweils diesen Ausschluß vornehmen zu können, wende ich seit 2 Jahren ein besonderes panoptisches Färbeverfahren in der Zählkammer an, s. A. f. O. Bd. 108). Auf' den Liquorbefund und die Temperatur. hin sofort Radikaloperation (Dr. Zausch). Sie ergab ein Cholesteatom, Granulationen in Gegend des ovalen Fensters und breite Einschmeizung der Wand des horizontalen Bogenganges, so da $\beta$ man in dessen Lumen hineinsehen konnte. Es wurde alles zur Labyrinthresektion bereit freigelegt, diese selbst aber aufgeschoben, weil eine Sinusblutung eintrat. Sofort nach der Operation wurde endolumbal nach der Linckschen Vorschrift bereitete $2 \%$ ige Vuzinkochsalzlösung in. Menge von $10 \mathrm{ccm}$ nach Abfließen- 
lassen der gleichen Liquormenge an der üblichen Stelle (dritter bis vierter Interspinalraum) injiziert.

Anderen Tages, etwa 12 Stunden nach der Radikaloperation, Labyrinthresektion nach Neumann (Operateur: Oberarzt Dr. Nühsmann); bei der Eröffnung des Labyrinths entleerte sich etwas trübe Flüssigkeit. Die Operation verlief im übrigen ohne Besonderheiten; der Meatus acusticus wurde nicht ganz erreicht. Nach der Operation sofortige Lumbalpunktion (dritte Punktion). Der unter $450 \mathrm{~mm}$ Wasser stehende. Liquor ist bereits markroskopisch eitrig, Zellzahl 2500 , davon $90 \%$ polymorphkernige Leukozyten; Bakterien ließen sich weder in der Kammer, noch in der Kultur nachweisen. Im Anschluß am die Lumbalpunktion Vuzineinlauf wie arn Vortage; Beckenhochlagerung für 6 Stunden; 3 g Urotropin täglich.

Am andern Tage deutliche klinische meningitische Zeichen. Trotz gut überstandener Operation verschlechterte sich das Allgemeinbefinden zusehends und schon am 3. Tage nach der Labyrinthresektion trat, nachdem noch zweimal vuzinisiert worden war, das Liquorbild aber immer bösartiger geworden war, und nachdem zuletzt auch kulturell Streptokokken erschienen waren, der Exitus ein.

Am Tage nach der Labyrinthresektion fand ich den frischen Verband kolossal mit Flüssigkeit getränkt; meine-Vermutung, daß es sich um LiquorausfluB von der Stelle des Labyrinthstumpfes handele, wurde mir vom Operateur bestätigt; ich begründete darauf den Plan, nachzuforschen, $o b$ in diesem den Verband tränkenden Liquor das Vuzin nachzuweisen sei. Außerdem beschloß ich, von nun an die Lumbalpunktate zu analytischen Versuchen zu verwenden. Ich versprach mir von der ersten Versuchsanordnung um so mehr, als ja bei der vorliegenden Liquorfistel am Felsenbein die Verhältnisse für eine retrograde Liquorströmung besonders optimal liegen mußten. Hier, an der Einfallspforte der Infektion, herrschte durch eine Art von Dauerdrainage ein negativer Druck und man konnte hoffen, daß auch im subarachnoidalen System ein Druckgefälle bestand; das vom Rückenmark her abfiel. Möglicherweise wurde in Verbindung mit der Beckenhochlagerung das Aufsteigen der Vuzinlösung gegen den inneren Gehörgang hin erheblich bèünstigt.

Zum Zweck des chemischen Vuzinnachweises setzte ich mich mit Herrn Professor Dr. Kochmann, Direktor des pharmakologischen Institutes, in Verbindung. Mit außerordentlicher Liebenswürdigkeit und großem Interesse hat er nicht nur durch Hinweis auf die Literatur, die ich von anderer Seite nur bruchstückweise hatte erhalten können, sondern auch durch tätige Unterstützung meine analytischen Versuche gefördert und mir ein selbständiges Weiterarbeiten ermöglicht: Auch an dieser Stelle spreche ich ihm meinen wärmsten Dank aus.

Bei der Untersuchung auf Vuzin kommt es darauf an, erstens überhaupt die Reaktionen auf dieses Alkaloid und ihre Empfindlichkeit zu kennen, zweitens eine Methode, die für den Nachweis gerade in Liquor geeignet ist, herauszufinden.

Archiv f. Ohren-, Nasen- u، Kehlkopfheillkunde. Bd. Io8. 
I. Vuzin ist chemisch ein Isoctyl-Hydrokuprein; es stellt einen Ester des Isoctyl-Alkohols mit dem chininverwandten Alkaloid Hydrokuprein dar. Prüfung unserer $10 \%$ igen Lösung mit Lackmuspapier zeigte saure Reaktion, auch der Geschmack war deutlich sauer. Seine Strukturformel ist unter Zugrundelegung der Chininformel von Skraup und Königs $(20,48)$ folgende:

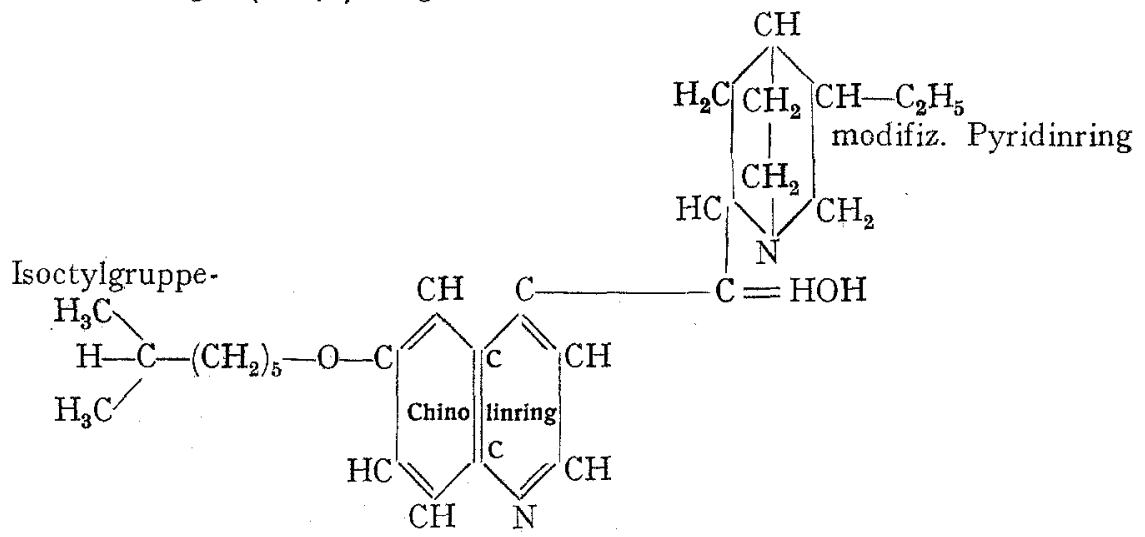

Der chemische Nachweis stützt sich darauf, daß Vuzin sich leicht in seine Hauptkomponenten spalten läßt, und diese dann nachgewiesen werden können. Zur Verfügung steht erstens der Nachweis des Isoctyloder Kapryl-Alkohols. Spaltet man $0,05 \mathrm{~g}$ Vuzin in $2 \mathrm{ccm}$ verdünnter Schwefelsäure unter Erwärmung, soll sich der außerordentlich charakteristische, wie bei allen höheren Alkoholen der Fettreihe fruchtähnlich-aromatische Geruch des Alkohols zeigen. Ich habe zunächst dieser: Reaktion keine besondere Beachtung geschenkt, später gelang mir der Nachweis, daß auch bis I mg Vuzin ( $1 \mathrm{ccm} \mathrm{I \%} \%$ iger Vuzinkochsalzlösung und $2 \mathrm{ccm}$ konzentrierter Schwefelsäure) mittels Geruchsprobe nachweisbar ist, und zwar haben fünf unvorbereitete Kontrollpersonen die Reaktion wahrgenommen. Immerhin wird man ihre Bedeutung in Anbetracht des individuell sehr verschieden scharfen Geruchvermögens. gering einschätzen.

Die Reaktionen auf dem Kupreinrest sind die gleichen, wie sie für das chemisch ganz nahe verwandte Chinin (Methylkuprein) Geltung haben. Die beiden charakteristischen und hochempfindlichen Reaktionen auf Chinin sind die sog. Thalleiochin- und Erythrochin-Reaktion, beides schöne Farbenproben. Über die Empfindlichkeit der ersteren finden sich in der Literatur verschiedene Angaben. Bylsma (1. c.), der sie bei Vuzin zuletzt ausgedehnt anwendete, fand sie noch bei einer Verdünnung des Vuzins von $\mathrm{I}: 20000$, d. i. $1 / 2 \mathrm{mg}$ in $\mathrm{Io} \mathrm{ccm}$ destillierten Wassers, positiv. Dagegen soll die Erythrochin-Reaktion nach demselben Autor noch in einer Verdünnung von I: rooooo, d. h. 1/10 $\mathrm{mg}$ 
in Io ccm Lösung, positiv ausfallen. Über die Technik der Reaktionen. siehe unten.

2. Wie ist das Vuzin aus den Verbandstoffen, wie aus dem Liquor in einer möglichst großen Menge zu gewinnen? Dieser Frage wurde die größte Beachtung und Sorgfalt zugewendet. Die Verbandstoffe blieben, nachdem sie den Liquor aufgenommen hatten, noch 3 Tage liegen, und erwiesen sich beim ersten Verbandwechsel am Tage des. Todes in den inneren Teilen auch etwas mit Blut durchtränkt. Zum geringen Teil. bestanden sie aus Jodoformgaze.

Der Verband wurde zunächst auf 8 Tage mit etwa $500 \mathrm{ccm}$ destillierten Wassers, das mit Salzsäure eben angesäuert worden war, digeriert, die braunrote Flüssigkeit darnach durchfiltriert zum chemişchen Nachweis verwendet. Nach Maßgabe seiner Löslichkeit mußte sich das Salzsäurevuzin in diesem Extrakt befinden. Über die zu erwartende Mengekonnten wir uns natürlich nur unbestimmten Vorstellungen hingeben. Wir hatten, wenn wir annehmen, daß die Liquorabsonderung in den. Verband nur an dem Tage der Operation erfolgte, bis zu diesem Termin $20 \mathrm{ccm} 2 \%$ iges Vuzin endolumbal appliziert, entsprechend einer Gesamtmenge von $4^{\circ} \mathrm{mg}$ reinen Vuzin. Nehmen wir an, das Vuzin habe sich im Liquor gleichmäBig verteilt! Eskuchen (3) gibt als Mittelwert für die gesamte im Zentralnervensystem frei zirkulierende Liquormenge $100 \mathrm{ccm}$ an, davon etwa $50 \%$ spinal, $20 \%$ epizerebral (Zysternen), $30 \%$ intrazerebral (Ventrikel). In der Annahme, da $B$ die Vuzinlösung nicht die Selbsterneuerungsbewegung des Liquor, die nach Eskuchen auf $6 \rightarrow 7$ mal pro Tag geschätzt wird, mitmacht, sondern, worauf sich unsere therapeutische Hoffnung gründet, im Liquor bleibt, kann man schätzen, daß sich die $40 \mathrm{mg}$ Vuzin in etwa $70 \mathrm{ccm}$ endolumbaler Flüssigkeit suspendiert finden werden. An die Möglichkeit, daß das Vuzin. schlecht resorbiert wird, kann man um so mehr denken, als seine Base nicht nur in der Kochsalzlösung ausfällt, sondern vielmehr noch im Liquor Fällungserscheinungen hervorruft (s. u.).

Angenommen, daß etwa Io $\mathrm{ccm}$ Liquor sich in den Verband hereingegossen, wären etwa 5-6 mgr Vuzin in der Digerierungsflüssigkeit: von $500 \mathrm{ccm} \mathrm{zu}$ erwarten gewesen, d. i. Mengen, die das 50-6o fache von dem darstellen, welches nach Bylsma noch eben durch Farbreaktion nachweisbar ist!

Zur Ermittlung des besten Isolierungsverfahrens wưrden zunächst einige Vorproben angesetzt, $\mathrm{zu}$ denen Ioo $\mathrm{ccm}$ verwendet wurden.

I. Vorprobe. $30 \mathrm{ccm}$ werden angesäuert und die ErythrochininReaktion angestellt; es entstand Eiweißfällung, kein Farbring.

2. Vorprobe. Weitere $30 \mathrm{ccm}$ werden zur Trockne eingedampft, der braune eiweißhaltige Rückstand mit wenig saurem Wasser unter kräftigen Reiben in der Reibschale extrahiert, das Filtrat mit Ammoniak 
leicht alkalisch gemacht und mit Chloroform ausgeschüttelt. Das Chloroform setzt sich schlecht $a b$ und wird unter Schwierigkeiten wieder gewonnen, abgehebert, verdampft, der Rückstand gelöst und auf Vuzin untersucht. Erythrochinprobe negativ.

3. Vorprobe. Der Rest der Ausgangsflüssigkeit wird alkalisch gemacht und mit Chloroform geschüttelt; die milchige Emulsion, die sich auf Ätherzusatz von der darüberliegenden, trüben, eiweißhaltigen Lösung nicht trennt, wird energisch zentrifugiert. Das nunmehr großenteils abgesetzte farblose Chloroform wird vorsichtig im Schälchen auf dem Wasserbade verjagt, der eigentümlich riechende kristalloide Rückstand zunächst mikroskopiert und als Fettsubstanz (?) erkannt. Alkaloidverdächtige Kristalle fanden sich nicht. Der Inhalt des Schälchens wurde nun in wenig angesäuertem Wasser aufgenommen und damit die Erythrochinreaktion angestellt. Sie erwies sich als negativ.

Bevor wir auf den Hauptversuch eingehen, sei das chemische Vorgehen begründet.

Das angewandte Isolierungsverfahren ist das bei Alkaloidnachweis übliche, nämlich Extraktion des Organs oder sonstigen Gegenstandes mit salzsaurem Wasser, Ausschüttelung der freien Base mit Chloroform, Äther, Benzol o. ä., nachdem die Lösung durch Ammoniak oder Natr. bicarbon. alkalisch gemacht ist. Nach Erwärmen auf dem Wasserbade bleibt das Alkaloid zurück, das, durch salzsaures Wasser von neuem in das lösliche salzsaure Salz überführt, nun dem Nachweis zugänglich ist. Während Bylsma empfiehlt, das Vuzin in alkalischer Lösung mit Äther auszuschütteln, haben wir auf Grund unserer Erfahrungen das Chloroform vorgezogen. Angesetzte Vergleichsversuche mit Chloroform und Äther als Ausschüttelungsmedien, angestellt mit Lösungen von Vuzinalkohol in destilliertem Wasser, in physiologischer Kochsalzlösung und in normaler Lumbalflüssigkeit, Reihenversuche, deren ausführliche Darstellung zu viel Raum einnehmen würde, zeigten nämlich, daß Chloroform aus derartigen Lösungen mehr Vuzin herausholt, als Äther. Ich habe auch ursprünglich die Thalleiochinreaktion heranzuziehen gedacht, doch zeigten Vorversuche in Übereinstimmung mit den Angaben anderer Autoren, daß diese Reaktion selbst bei korrektester Ausführung sehr viel weniger empfindlich ist, als die Erythrochinreaktion. Der Vollständigkeit halber sei erwähnt, daß dieselbe folgendermaßen angestellt wird: zur Chininsalzlösung gibt man Chlor- oder besser Bromwasser im Verhältnis I : 5, schüttelt und setzt sofort Ammoniak im Überschuß zu; es entsteht eine smaragdgrüne Färbung, bei hohem Prozentgehalt ein dunkelgrüner Niederschlag, der sich in Chloroform leicht löst. Als Grenzwert für die Reaktion fand ich die Verdünnung $I$ : 16000 , dagegen für die Erythrochininreaktion einen Titer von $I: 500000$ und $z$ war in 
gleicher Weise für Lösungen in destilliertem Wasser und Kochsalz; Bylsma fand für die letzte Reaktion I: rooooo. Die Technik der Reaktion, wie sie sich durch größere Versuchsreihen für meinen Zweck des Vuzinnachweises im Liquor als zweckmäßig herausgestellt hat, ist folgende: Io $\mathrm{ccm}$ Ausgangslösung + $0, \mathrm{I} \mathrm{ccm}$ Eisessig werden vermischt, dazu zwei Tropfen Bromwasser, schnell umschütteln bis wieder Farblosigkeit eintritt; ohne Verzögerung zwei Tropfen Io\% iger Ferrozyankaliumlösung dazugeben und bis zum Erreichen einer gleichmäßig hellgrünen Färbung schütteln; das Gemisch darf nicht mehr nach Brom riechen; nun sofort Überschichten mit $2 \mathrm{ccm}$ Ammoniakflüssigkeit. Bei positivem Ausfall bildet sich ein mehr minder breiter und intensiver violettroter Ring an der Oberfläche, bei schwacher Reaktion mit verwaschener Grenze in die darunter befindliche grüne Schicht übergehend. Bei I : I00 o00 zeigt sich meist nur ein Gelb- bzw. Farbloswerden der oberen Schicht der Mischung. Beim Schütteln wird das ganze Gemisch entweder gelblich oder violett. Sehr verfeinert wird die Reaktion, wenn man sofort nach Auftreten dẹs Farbstoffes, der von Fühner als Oxychinolin angesehen wird, mit I ccm Chloroform kräftig ausschüttelt, das sich violett gefärbt absetzt, während die darüberliegende Schicht die ursprüngliche grüne Farbe wieder annimmt. Diese Form der Reaktion ist so scharf, daß sie sich mir zur kolorimetrischen quantitativen Bestimmung von Vuzin als sehr geeignet erwies: Allerdings blaßt in einigen Stunden bei diffusem Tageslicht der violette Farbstoff ab, so daß man sich keine Farbenskala für dauernde Vergleichszwecke herstellen kann. Sehr zu beachten bei Anstellung der Erythrochininreaktion ist, daß zwischen ihren einzelnen Phasen keine Pausen entstehen dürfen, da sonst sicher vorhandenes Vuzin wenig oder gar nicht zum Nachweis kommt.

Diese technischen. Einzelheiten, das Ergebnis zahlreicher Vorversuche, habe ich für den Hauptversuch nutzbar. gemacht. Bévor ich ihn jedoch darstelle, seien noch andere Ergebnisse erwähnt, die sich beim Experimentieren mit Vuzinlösungen und normalem Liquor zeigten. Das Einbringen einer $I-2 \%$ igen Vuzinkochsalzlösung in den Liquor führt zu einer intensiven Trübung desselben. Während eine aus $10 \%$ igem Vuzinalkohol hergestellte Lösung von Vuzin in destilliertem Wasser in Verdünnung $I$ : Io ooo klar ist und bläulich fluoresziert (charakteristische Fluoreszenz der Chinalkaloide), ist eine gleich starke Kochsalzlösung trübe. Noch trüber ist eine gleiche Lösung von Vuzin in Liquor. Nach zweimal 24stündigem Stehen einer Lösung von Vuzin in destilliertem Wasser I : I0000 ist die Fluoreszenz fort, und die Lösung wird vuzinfrei befunden. Das Alkaloid haftet vielmehr den Glaswandungen an. Nach Bylsma fällt in der Kochsalzlösung die freie Base aus. Bylsma glaubt nun, daß im Serum, welches ähnliche Fällungserscheinungen dar- 
bietet, das Gleiche stattfindet, eine feste Eiweißbindung aber nicht erfolgt. Mit Rücksicht auf den Unterschied zwischen dem Verhalten der Kochsalz- und Liquorlöslichkeit kann man annehmen, daß Alkaloid auf die Eiweißsubstanzen eine gewisse Wirkung ausübt, die zur Fällung führt. Dabei handelt es sich wohl um eine lockere Bindung (Adsorption) des Vuzins durch das Eiweiß. Daß beim Schütteln mit Äther die Trübung sich aufhellt, ist ein Zeichen leichter Trennbarkeit der Verbindung. Für die Annahme adsorptiver Bindung spricht auch, daß das Vuzin sich mit der Erythrochininreaktion, unmittelbar am Liquor angestellt, nicht nachweisen läßt; es muß erst aus seiner Eiweißverbindung losgelöst werden.

Hauptversuche. I. $200 \mathrm{ccm}$ Ausgangsflüssigkeit werden gemäß Vorprobe 3 verarbeitet. Die Farbreaktion erwies sich als völlig negativ. 2. IoO $\mathrm{ccm}$ wurden gemäß Vorprobe 2 mit Chloroform, Ioo ähnlich mit Äther verarbeitet. In beiden Proben konnte kein Vuzin nachgewiesen werden.

Ergebnis: Bei der hohen Empfindlichkeit der Erythrochinreaktion mußte, die Einwandfreiheit des analytischen Verfahrens vorausgesetzt, - und daß es einwandfreie Resultate zu liefern vermag, davon habe ich mich an reichlichen Kontrollproben mit Liquor im Reagenzglas überzeugt, - Vuzin im Liquor gefunden werden können. Da die Liquorrhoe sofort nach der zweiten Vuzininjektion während der Zeit der Beckenhochlagerung erfolgte, konnte angenommen werden, daß, wenn überhaupt das Vuzin bis zum Endokranium gelangte, es in nachweisbaren Mengen dem ausströmenden Liquor beigemengt sein mußte. Daß andererseits Liquor außerhalb des Körpers Vuzin nicht zu zerstören vermag, habe ich (s. u.) ebenfalls nachgewiesen. Dennoch wurde nicht einmal $1 / 50$ mgr Vuzin gefunden.

Wir sind, obwohl dessen bewußt, da $B$ nur ein positiver Befund eine eindeutige Entscheidung gebracht hätte, immerhin zu der Annahme berechtigt, da $\mathrm{B}$ in diesem Falle das Vuzin den Weg, den wir ihm weisen wollten, nicht gegangen ist. Wir können nicht mit Sicherheit sagen, aus weIchem Grunde und wohin es sonst etwa gelangt ist. Die Strömungsverhältnisse des Liquor und die endolumbale Resorptionsfähigkeit für die VuzineiweiBverbindung sind uns unbekannte Faktoren, denen wir weiter nachzugehen als unsere nächste Aufgabe betrachtet haben.

Die bei den letzten Lumbalpunktionen jeweils gewonnenen Liquores konnte ich erst aus äußeren Gründen analysieren, als sie schon ca. 8 Tage bei Zimmertemperatur gestanden hatten. Die Punktionen waren jedesmal in etwa 24 stündigem Abstand vorgenommen worden. In sämtlichen Punktaten fand ich kein Vuzin, glaubte aber zunächst, das Ergebnis deswegen nicht bewerten zu können, weil vorhandenes Vuzin während 
des Stehens hätte verändert worden sein können. Spätere Versuche (s. u. Fall 2) haben mich indessen belehrt, daß in einer künstlich hergestellten Vuzin-Liquorlösung das Vuzin noch nach mehreren Tagen fast genau quantitativ nachweisbar ist.

Wir können aus unseren Ánalysen das Fazit ziehen, daß I. $20 \mathrm{mg}$ endolumbalinjizierten Vuzins nach 24 Stunden im Liquor der Injektionsstelle nicht mehr nachweisbar sind, daß 2. die physiologische Liquorströmung trotz Kopftieflagerung und trotz kranialer Liquorfistel das Aszendieren der Vuzinlösung nach der Schädelhöhle hin nicht begünstigt hat. In diesem Falle muß der effektive Wert der endolumbalen Vuzinbehandlung gleich null gewesen sein.

Die Bestätigung dessen ergab der weitere Verlauf. Die fortgesetzte Vuzinisierung hat der Ausbreitung der Erreger in kraniokaudaler Richtung keinen Einhalt zu tun vermocht. Die letzte Lumbalpunktion ergab Streptokokken, die Sektion ließ an der Konvexität und der Basis des Hirns sowie in der Gegend der Brücke, des Kleinhirns und des verlängerten Markes eitrige Infiltration der Maschen der weichen . Hirnhäute erkennen; die Rückenmarkshäute waren getrübt und zeigten besonders im kaudalen Abschnitt eitrigen Belag. Im Eiterausstrich wurden Streptokokken gefunden.

Der hier intra vitam geführte Nachweis, daß eine in den Subarachnoidalraum-in Gegend der Lendenwirbelsäule injizierte Lösung einer körperfremden Substanz trotz a priori für die Aszendenz günstiger Bedingungen nicht in das Endokranium gelangt ist, dürfte der Literatur zufolge nicht häufig geführt worden sein. Zwar sind an menschlichen Leichen und an lebendem Säugetier von verschiedenen Autoren Versuche zu dem Ende gemacht worden, die Verteilung endolumbaleinverleibter Stoffe im Liquor zu prüfen. Die Befunde Goldmanns und Jacobs (zit. bei 30), daß bei Tieren injizierte Farblösungen bis zur Hirnoberfläche gelangen, ergänzte Fleischmann durch Versuche an Katzen und Kaninchen dahingehend, daß vorzugsweise die Hirnbasis umspült wird. An menschlichen Leichen kam Jochmann zu analogen Schlüssen (30). Beide Versuchsanordnungen mit ihren Ergebnissen vermögen indessen darüber nichts auszusagen, ob unter physiologischen Umständen die Diffusionsverhältnisse im Liquor des Menschen entsprechend sind. Daß vielmehr ein Schluß aus dem Verhalten des tierischen Subarachnoidalraumes auf den bei Menschen schon aus bestimmten anatomischen Gründen nicht zulässig ist, 'hat bereits Walter (30) gezeigt. Bei der Leiche sind die Verhältnisse naturgemäß gänzlich anders als beim Lebenden: statt der verschiedenen Bewegungsvorgänge (Pulsationen, Strömung, Diffusionen), die im Leben einen artifiziellen Aufstieg von Stoffen fördernd odẹr vielleicht eher hindernd beeinflussen mögen, herrscht Stase; statt Überdruck ein 
Gleichgewichtszustand um $760 \mathrm{~mm}$; die Turgorverhältnisse der Wände sind anders; der Liquor selbst hat physikalische ev. sogar tiefgreifende chemische Änderung erfahren. Wichtiger sind zur Beurteilung der normalen Physiologie die Beobachtungen der Chirurgen bei Anwendung der endolumbalen Tropakokainanästhesie nach Bier; bekannt ist, daß man diese nicht nur durch Variierung der injizierten Menge und Konzentration (Höhe des injizierten Flüssigkeitszylinders), sondern auch durch geeignete Körperlagerung(Beckenhebung) kranialwärts ausdehnen kann, derart, daß aus unliebsamer zu starker Diffusion gegen die Oblongata hin schon Todesfälle vorkamen. $\mathrm{Zu}$ bedenken ist allerdings, daB es sich hier um Substanzen handelt, die infolge ihrer Lipoidlöslichkeit eine ausgesprochene Affinität zu den Zentralnervensystemselementen besitzen. Erinnert sei daran, daß Gennerich (29) seine Méthode der endolumbalen Salvarsantherapie mit Beckenhochlagerung auf die Hoffnung automatischer Aszendenz aufbaut; die Tatsache, daß der Wert dieses Verfahrens von erfahrenen Autoren gering angeschlagen wird, da die Erfolge nicht besser sein sollen; als bei extràlumbaler Salversananwendung, ist vielleicht zum Teil darauf zurückzuführen, daB aben die Grundlage nicht sicher ist.

Wir haben bisher nur die physiologischen Verhältnisse berücksichtigt. Wie ist es bei Vorliegen meningitischer Veränderung? Wir müssen annehmen, daß bei lediglichen Reizzuständen der Meningen durch angrenzende Prozesse regionäre Hyperämie, leichtes Gequollensein, geringe Infiltration der Gefäßscheiden, kurz Veränderungen vorliegen, die im allgemeinen das Lumen der Pialräume wenig beengen werden. Anders bei vorgeschrittenen eitrigen Stadien; hier findet der Obduktor außer eitriger Infiltration entlang den Venen diffuse Eiterplaques im Balkenwerk der Spinnwebenhaut, ev. plastisches; sulziges Exsudat, fibrinöse Stränge, breite Verbackungen. Bekanntlich kommt es zu solchen Veränderungen am weitgehendsten bei der tuberkulösen, dann bei der gewöhnlichen eitrigen; am wenigsten oft bei der epidemischen Meningitis. Solche Kommunikationsstörungen müssen den Aufstieg von Medikamenten erheblich erschweren. Fleischmann (1. c., S. 340) hat meines Erachtens recht, wenn er die Versuchsergebnisse von Schöne (zit. 30), der bei an epidemischer Meningitis im Sterben liegenden Kindern endolumbalinjiziertes Methylenblau bis zum Kopfmark und zur Hirnbasis aufsteigen sah, als für pyogene Meningitiden nicht ohne weiteres geltend anspricht. Den Schluß, zu dem er auf Grund seiner drei Behandlungsversuchen mit Eukupin sowie der zitierten Ergebnisse von Wolffs (zit. n. 30) bekannter endolumbaler Protargolbehandlung kommt, da $B$ nämlich ,eine Verteilung intralumbalinjizierter Lösungen über die Rückenmarks und Schädelhöhle bei bestehender Meningitis auch nicht mit der Einschränkung zu er- 
warten sei, daß die Konvexität des Gehirns so gut wie nicht erreicht wird", vielmehr wesentlich schlechter sein und je nach dem Grad des Hindernisses mehr minder große Abschnitte des Rückenmarkskanales betreffen muß, können wir bestätigen. Bei der Sektion einer otogenen Meningitis vor Jahren mußten wir sehen, daß endolumbalinjiziertes Dispargen trotz Kopftieflagerung nur bis zur Oblongata aszendiert war; dasselbe sah Eskuchen (29, S. I76) bei Methylenblauanwendung. Andererseits fand $\mathrm{K}$ äding (55) bei einem Fall von tuberkulöser Meningitis injiziertes kolloidales Silber an der Hirnbasis wieder. Die Verhältnisse sind also doch von Fall zu Fall verschieden. Während das Vorliegen von Hydrozephalus im allgemeinen den geringsten Einfluß ausüben dürfte, wird man in der Annahme nicht fehlgehen, daß Kommunikationsstörungen durch plastisches Exsudat auf der einen, die Resorptionsverhältnisse des angewandten Mittels auf der anderen Seite ceteris paribus die Verteilung bestimmenden Faktoren darstellen. Erstere werden sich nicht immer mit völliger Sicherheit graduell feststellen lassen; sie werden daher in der Praxis in zweiter Linie zu berücksichtigen sein. Das wichtigste sind zweifellos die Resorptionsverhältnisse.

In unserem Fall ist das Vorliegen des ersten Faktors nicht anzunehmen. Die erste Vuzininjektion fand im Stadium der initialen diffusen Meningitis, die zweite im Beginn des eitrigen Stadiums statt, die Lumbalpunktionen ergaben stets Liquor; die Sektion - Tod am vierten. Tage nach Beginn der Meningitis - zeigte, da $B$ besonders die Spinalräume nicht so affiziert waren, daß völliger Abschluß in vivo anzunehmen wäre. Unser Fall scheint also in der Richtung. Fleischmanns These zu ergänzen, daß trotz bestehender Kommunikation wohl die entgegengerichtete gesteigerte Strömung des Liquor das Medikament am Aufstieg verhindert hat.

Ich hatte die Arbeit so weit beendet, als ein zweiter Fall mir Gelegenheit bot, dem Sonderfaktor der Resorption noch weiter nachzugehen. Er ist in Kürze folgender:

Der 12 jährige Hans B. kommt mit deutlichen zerebellaren und meningitischen Zeichen herein. Die klinische Diagnose auf linksseitigem Kleinhirnabszeß im Gefolge von Cholesteatomeiterung wurde zunächst durch die Lumbalpunktion ergänzt; es fand sich unter $420 \mathrm{~mm}$ Druck stehender, opaleszenter, Pandy positiver Liquor mit etwa 300 , davon $27 \%$ polymorphkernigen, Zellen im cmm; in Kammer, Ausstrich und Kultur keine Bakterien; geringer Eiweißgehalt, schwache Moritzsche Probe, gute Reduktionskraft für FehlingHaine ließ im Verein mit den vorigen an einem kleinen, unschriebenen, aber intensiv gereizten Meningealbezirk in Gegend des Abszesses denken.

Die sofortige Radikaloperation mit breiter Freilegung der hinteren Schädelgrube (Dr. Lederer) bestätigte vollauf die Diagnose durch den Befund eines eigroßen, jauchigen Kleinhirnabszesses der linken Hemisphäre. Sofort prophylaktische Vuzinisierung, und zwar mit je to $\mathrm{ccm} \mathrm{2,} \mathrm{nicht} 1 \%$ oiger Lösung. 
Zum Zweck der Nachforschung nach Verbleib und Verteilung des Alkaloides wurden nun 8 Stunden nach der ersten, I2 Stunden nach der zweiten und etwa 20 Stunden nach der dritten Injektion je Io ccm Liquor der Untersuchung auf Vuzin mit den oben geschilderten Verfahren unterzogen. Ausreichende Mengen von auf Eis aufbewahrtem normalem Standardliquor sowie von solchem frischen Vuzinliquor standen zur Kontrolle zur Verfügung. Von dem zu prüfenden Liquor wurden jeweils sofort

je $5 \mathrm{ccm}$ mit Äther,

je $5 \mathrm{ccm}$ mit Chloroform unter den beschriebenen Kautelen verarbeitet. Dazu kamen je zwei Kontrollen

je $5 \mathrm{ccm}$ in vitro vuzinisierter Liquor,

je 5 ccm sicher vuzinfreier Normalliquor.

Endlich wurde stets eine frische kolorimetrische Titerreihe von Vuzinlösungen I: 10000-I : 500000 mittels der Chloroformausschüttelungsmodifikation der Erythrochinprobe hergestellt.

Es ergab sich nun, sowohl für den 8 , sowohl 12 wie den 20 stündigen Liquor:

I. keine Spur Vuzin, weder mit dem Chloroform noch Ätherverfahren;

2. Vuzin, in vitro dem Liquor zugesetzt, ließ sich stets fast quantitativ nachweisen;

3. auch nach mehr als 24stündigem Stehen ist aus künstlichem Vuzinliquor I : roooo das Aikaloid fast restlos zu gewinnen, wird also im Liquor in vitro nicht verändert.

Wir haben aus dem zweiten Teil der Versuchsreihe im Fall I, sowie durch die Versuche im Fall 2 den Beweis erbracht, daß im Einzelfall schon 8 Stunden nach der Injektion jede Spur von Vuzin von der Intima des Subarachnoidalraumes gebunden, bzw. zerstört oder weitergegeben worden ist. Bevor ich jedoch die Ergebnisse der ganzen Versuche in ihrer Bedeutung zur Frage der Wirksamkeit der Vuzintherapie zusammenfasse, noch einige Worte: erstens zur Frage der Resorptionsfähigkeit des arachnoidalen Endothels überhaupt, zweitens zur Frage der Resorption und Ausscheidung des Vuzins an anderen Organen.

Zum ersten Punkte liegen experimentelle Arbeiten von Ziegler (Iog), Lewandowsky (68), Cavazzani (2I) für endolumbal applizierte Ferrozyan- und Jodsalzlösungen, von Tuffier (IOO) und KrönigGauB (62) für Kokain und Stovain, von Hohns (45) und Holzmann (49) für Serumarten vor. Ihr allgemeines Ergebnis ist, daß, zum mindesten unter physiologischen Umständen und am Versuchstier, die Innenhaut ein großes Eliminationsvermögen für gelöste Fremdsubstanzen zeigt. An sich ist diese Tatsache gerade bei den angeführten Substanzen erklärlich; Narkotika als spezifisch lipoidaffine Stoffe, ebenso Serumeiweiße gehen auch den umgekehrten Weg vom Blut in den Liquor relativ leicht (s. oben); für Ferrozyankalium und Jodsalze ist dasselbe von einigen Autoren beobachtet worden. Nun wissen wir aber, daß der krankhaft veränderte Liquor sich außerordentlich schnell erneuert, so daß Grund zu der Annahme vorliegt, daß lösliche Fremdsubstanzen 
auch anderer Zusammensetzung, als die eben beschriebenen, leicht herausgeschwemmt werden.

Uber die Resorptionsgeschwindigkeit gelösten Vuzins in tierischen Geweben finde ich, ebensowenig wie für seine Homologen, in der so reichhaltigen Literatur über die Chininderivattherapie genaue Angaben, nicht einmal in den Arbeiten über Tiefenantisepsis nach Klapp. Von den Chininsalzen selbst gilt die Regel, daß sie mit Ausnahme der freien Alkaloide und der Tannate vom Verdauungskanal aus schnell resorbiert, von den Körperzellen schnell zerstört und zum geringen Teil unverändert ausgeschieden werden; ihrer subkutanen Anwendung (ohne Urethan, Antipyrin u. ä.) steht die Neigung zur Entstehung von örtlichen Nekrosen entgegen. Bei intramuskulärer und intravenöser Injektion erfolgt die Verteilung außerordentlich schnell (37), für praktische Zwecke oft zu rasch. DaB über die Resorbierbarkeit der modernen Chininund Hydrokupreinderivate so wenig sicheres bekannt ist, ist um so erstaunlicher, als ihre Lösungen, vom innerlichen Gebrauch abgesehen, in größeren Mengen in Körperhöhlen infundiert wurden; ich erinnere an Füllung von Gelenkhöhlen, von Abszessen (Mastitis), von Pleuraempyemhöhlen und Peritonealsack, worüber mehrere Arbeiten aus der Kriegszeit vorliegen. Als Zeichen einer schnellen Resorption kann man vielleicht das verschiedentlich beschriebene Auftreten hoher Fieber- reaktionen nach derartiger Anwendung deuten (89).

Klapp $(57 \mathrm{c})$ nahm bei der Wundumspritzung ein Verbleiben des Vuzins im Gewebe von I2-24. Stunden an, vertrat im übrigen' die Anschauung, da $B$ es weniger durch Resorption, als durch Ausscheidung in die Wundfläche entfernt wird. Einen Beweis hierfür hat, soviel ich sehe, kein Autor zu führen unternommen. Sichtbar soll der Vuzinwall bei Umspritzungen, so z. B. auch bei Erysipel, bei dem u. a. Ostrowski (78) gute Erfolge sah, noch viel kürzere Zeit sein. Frühzeitig wandte man sich daher der Kombination mit Nebennierenpräparaten und Kolloiden, wie Gummi arabikum (2) zu. Zu Schlüssen, die den Vorzug größerer Objektivität haben, aber den zitierten praktischen Erfahrungen zuwiderlaufen, kommt Bylsma (1. c.) auf Grund von exakten chemisch-analytischen Versuchen am Tier. Er injizierte Mäusen in jedes Hinterbein je $1 / 2 \mathrm{ccm} \quad 3 \%$ ige Vuzinkochsalzlösung (also $3 \mathrm{mg}$ !) subkutan und intramuskulär, amputierte sie in verschiedenen Zeitabständen und führte mittels der Erythrochinreaktion quantitative Analysen auf Vuzin aus. 24 Stunden post injektionem fand er noch ansehnliche Mengen, nach 4 mal 24 Stunden noch Reste im Gewebe vor. Bei intravenöser Injektion nahezu tödlicher Dosen bei Meerschweinchen zeigte sich dagegen schnelle Verteilung und Zerstörung des Giftes, vielleicht in der Leber. Weder bei subkutaner noch intravenöser Gabe subletaler Dosen konnte Bylsma Vuzin im Harn nachweisen. Nach 
Böcker (I3) wird dagegen Vuzin durch Nieren und Darm, wenn auch sehr langsam, ausgeschieden.

Wir können also sagen, daß der von uns geführte Nachweis des sehr schnellen Verschwindens von Vuzinlösungen aus dem Lumbalsack bei Meningitis im Einklang steht mit den Verhältnissen, deren Geltung für andere lösliche Fremdsubstanzen nachgewiesen worden ist. Die Eliminierung erfolgt wahrscheinlich schneller als bei subkutaner Anwendung.

Ich möchte nicht schließen, ohne einen Fall gebracht zu haben, bei dem die Vuzinbehandlung ergebnislos war.

Fall 3. S., Hilma, 4 Monate. Aufnahme I. VI. 21.

Kam zur Aufnahme mit akuter Otitis und Mastoiditis links, die am Ir. VI die einfache Aufmeißelung notwendig machte. Bei der Operation wurde der in Granulationen vollkommen eingebettete Amboß mit entfernt; am I5. VI. begannen meningitische und vestibulare Reizsymptome sowie Temperaturanstieg. Die Lumbalpunktion ergab: Anfangsdruck $450 \mathrm{~mm}$. Aussehen und Farbe: klar, in dicker Schicht leicht trübe. Pandy nativ +++ . Zellen 525, davon $94 \%$ polymorphkernige, in der Zählkammer außerdem massenhaft Streptokokken in kurzen und langen Ketten.

[Anmerkung. Ich möchte noch hervorheben, daß in Fall I und 3 in der Zählkammer mittels vitaler Methylgrünpyroninfärbung bereits die Erreger gefunden wurden, welche sich rotviolett färbten. Diese Beobachtung hatte ich bis zur Fertigstellung meiner Arbeit (I2) noch nicht machen können].

Danach wurden $10 \mathrm{ccm} 2 \%$ iges Vuzin injiziert.

Am I6. VI. Labyrinthoperation (Oberarzt Dr. Nühsmann). Es fand sich Defekt des Steigbügels am ovalen Fenster, aus dem sich etwas Eiter entleerte. Bei der Labyrinthresektion zeigte sich auch am inneren Gehörgang etwas Eiter. Eine vor der Operation vorgenommene Lumbalpunktion hatte keinen Liquor ergeben. Daher wurde von zweiter Vuzinisierung abgesehen.

Das Kind kam am selben Abend zum Exitus und die Obduktion ergab eine ausgebreitete eitrige Meningitis der Konvexität und der Basis, Erfüllung der erweiterten Ventrikel mit etwaș trübem Liquor. Im Ausstrich der Pia fanden sich Streptokokken.

Epikrise: Eine schwere traumatische labyrinthäre Meningitis blieb durch eine einmalige Vuzininjektion unbeeinflußt; daß aus diesem Fall allein weitreichende Schlüsse nicht zu ziehen sind, dessen bin ich mir wohl bewußt.

$$
\text { Zusammenfassung. }
$$

I. . Die Behandlung der ausgebreiteten otogenen Meningitis ist nach allem und trotz allem, was darüber geschrieben wird, eine crux medicorum. Bei genauer Prüfung der beschriebenen geheilten Fälle kann man sich des Eindrucks nicht verschließen, daß das post hoc und propter hoc kaum jemals sicher auseinandergehalten wurden. Die Polypragmasie in der Meningitisbehandlung in dem internistischen Grenzgebiet deutet auch nicht darauf hin, das bis jetzt Aussicht bestände, ein einigermaßen spezifisches Mittel in die Hand zu bekommen. 
2. Will man die Einwirkung eines Medikamentes auf die Meningitis objektiv prüfen, so muß man den. Bakteriengehalt des Liquor als Kriterium nehmen und im übrigen das Verhalten des Arzneimittels im Liquor kontrollieren.

3. In zwei beobachteten Fällen schwerer bakterieller Streptokokkenmeningitis erwies sich das Vuzin als nutzlos.

4. Es wurde nachhgewiesen, daß das Vuzin schon 8 Stunden nach der Injektion aus dem Liquor verschwunden ist, wahrscheinlich nicht durch Zerstörung, sondern durch Resorption. Diese Tatsache wird durch den Vergleich mit anderen Substanzen und mit der Resorptionsgeschwindigkeit des Vuzins an anderen Organen bestätigt.

5. Es ist nicht wahrscheinlich, da $B$ das Vuzin endolumbal eine desinfizierende Wirkung ausübt, und zwar, weil es zu kurze Zeit in der wirksamen Konzentration vorhanden war. Nehmen wir mit Bylsma an, die Desinfektionskraft auch für Streptokokken ( $1: 80000$ ) werde schon durch Kochsalzgehalt auf $1 / 3$, durch Zellgehalt des Liquor nochmals um $2 / 3$ und endlich durch den Eiweißgehalt herabgesetzt, so läge der Titer unserer endolumbalen Vuzinkonzentration I:5000-I: I0000 tatsächlich bestenfalls bei $I: 25000$ und $I: 50000$. Zeitlich gleichförmige Resorption in genau 8 Stunden gesetzt, wäre der Grenztiter in 3-6 Stunden erreicht; erfolgt die Resorption im Anfang schneller als später, liegt der wirksame Titer noch früher. Werden daher $\mathrm{klinische}$ Wirkungen von endolumbaler Vuzinapplikation beobachtet, so muB man sie anderen, nicht aber desinfizierenden Einflüssen zuschreiben.

6. Es ist, wie sich gezeigt hat, sehr schwer, bei Meningitis endolumbalapllizierte gelöste Substanzen an den Ort der Einfallspforte im Endokranium zu dirigieren, selbst wenn ein Kommunikationshindernis nicht besteht und das Aszendieren des Medikamentes durch Sonderbedingungen noch so begünstigt erscheint. Hinderlich ist wahrscheinlich die bei Meningitis beschleunigte kraniokaudalwärts gerichtete Liquorströmung.

7. Es wäre berechtigt, die Versuche einer subarachnoidalen Durchspülung in lumbokranialer Richtung mit Vuzin in schweren aussichtslosen Fällen wieder aufzunehmen, da bei dieser Anordnung die nachgewiesenen Nachteile der bisherigen Technik doch wohl bis zu einem gewissen Grade vermieden werden. 


\section{Literatur.}

I. Alt, Mon. f. O. n I9I2.

2. Ansinn, M. m. W. I9I8, 20.

3. Arnoldson, Internat. Zentr. f. O., Bd. XIV, S. 225.

4. Bardachzi, Med. Kl. I920, 5.

5. Barr, zit. nach Fleischmann.

6. Barth, Arch. f. klin. Chir., Bd. I05.

7. Berggren, Internat. Zentr. f. O., Bd. XIII, S. 107.

8. v. Bergmann. Chirurg. Behandlg. d. Hirnkrankheiten, 2. A., S. 187.

9. v. Bergmanñ-. Küttner-Krönlein, Handb. d. prakt. Chir., 4. A. 1913, S. 177 u. 207.

Io. Bieh1, Arch. f. O. I9I9, I04.

I I Bier, Hyperämie als Heilmittel, 6. A., I907, S. $382 \mathrm{f}$.

I 1 a. - D. Ztschr. f. Chir., Bd. 5I, S. 3or.

I2. Birkho1z, A. f. O., Bd. I08.

I3. Boecker, D. m. W. I920, 37.

I4. Bönninghaus, Die Meningitis serosa acuta, I897, S. $67 \mathrm{f}$.

I5. Bondy, Mon. f. O. IgI6, S. 493.

I5 a. - W. kl. W. I9I7. Zur Frage der Heilbarkeit....

16. Borchard-Schmieden, Lehrb. der Kriegschirurgie.

I7. Borries, Internat. Zentr. f. O., Bd. XV, S. 188.

I7 a. - ebenda, Bd. XIV, S. 252.

I 7 b. Zeitschr. f. O., Bd. 76.

18. Braun-Husler, D. m. W. I9I2, 38.

19. Brieger, Verh. d. otol. Ges. I899.

rga. - Verh. d. otol. Ges. I9I2.

20. Bylsma, Zeitschr.f. d. ges. experim. Medizin; XI, 5/6. Die pharmakol. Wirkung von Vuzin und Eukupin.

21. Cavazzani, Zentralbl. f. Physiol. 1892.

22. v. Caneghem, Verh. d. otol. Ges. 19I2.

23. Coglievina, W. kl. W. I9I6, 36 .

24. Cohn, Zeitschr. f. O., Bd. XXXVIII.

25. Denk u. Leischner, Verh. d. Chir. Kongr. I9II.

26. Denker, Verh. d. otol. Ges. Igr2.

26a. - Zeitschr. f. O., Bd. LXX.

26b. - u. Brünings, Lehrbuch, 4/5. A., I920.

27. Döderlein, Zeitschr. f. O., Bd. LXXVII, S. I4.

28. Eden, D. Zeitschir. f: Chir. I9I8.

29. Eskuchen, Die Lumbalpunktion 1919.

30. Fleischmann, Pass. Beitr. I9I8, X.

3I. Friedemann, Berl.. kl. W. I9I6, 16.

32. Gerstmann, W. kl. W. I919, 62.

33. Goetz-Hanfland, D. m. W. I9I6.

34. Goording, Intern. Zentr., Bd. XV, S. 245.

35. Gradenigo, Arch. f. O., Bd. 47.

36. GroBmann, Pass. Beitr. I918, X, S. 398.

37. Hartmann u. Zila, Arch. f. exp. Path. u. Pharm. I9r8, 81.

38. Henle, Mitt. aus den Grenzgeb. der Medizin u. Chirurgie I896, Bd. I. 
39. Henschen, im Handb. d. ges. Therapie (Penzoldt-Stintzing), 4. A., I9IO, Bd. 4, S. 574 u. $58 \mathrm{I}$.

40. Heine, Operationen am Ohr, 3. A., I9I3, S. 224 u. 227.

4I. Heymann, Internat. Zentr. f. O. IgII.

42. Herrschell, M. m. W. Igr2, 39.

43. Hinsberg, Deutsche otol. Ges. I9I2.

44. His, Zeitschr. f. ärztl. Fortbildung I920, I5.

45. Hohns, zit. nach. Levy.

46. Holmgren, Internat. Zentr. f. O., Bd. XIV, S. 2 I.9.

47. - Internat. Zentralbl. f. O., Bd. XIII, S. 107.

48. Holleman, Organ. Chemie, Io. A.

49. Holzmann, Neue d. Chir., Bd. XII.

50. Ibrahim, Med. Kl. I910, 48.

5I. Isemer im Handb. von Katz u. Blumenfeld, 3. A., Bd. I, 2. Hälfte.

52. Jacob, Berl. ki. W. I8g8.

53. Jansen, zit. nach Koerner.

54. Jansen-Kobrak, Prakt. Ohrenheilkunde.

55. Kaeding, Med. K1. I920, 39.

56. Kafka, Zeitschr. f. Neurol. u. Psych. I9I3, I5.

56a. - Mon. f. Neurol. u. Psych. I909, 24.

56b. - Zeitschr. f. d. ges. Neurol. u. Psych. I9I2, 9.

57 a. Klapp, M. m. W. 1915,5 .

57 b. - M. m. W. 1918, S. 497.

57 c. - D. m. W. Igr7, S. I 80 .

58. Knapp, Intern. Z. f. O,, Bd. III, S. 82.

59. Knick, Verh. d. otol. Ges. IgI3.

59a. - Verh. d. Otol. Ges. I9I2. Discuss. zu (22).

6o. Kobrak, Th. d. Gegenwart 19I6, V.

6I. Körner, Otitische Erkrankungen I908, 4. A., S. 58.

61 a. - dgl. Nachträge, S. 72 u. 76.

62. Krönig-GauB, zit. nach Fleischmann

63. Landsberger, W. m. W. I9I6, 45 .

64. Lenhartz, Vers. d. Naturf. u. Ärte I895, ref. M. m. W. I895, S. 942.

65. Lehmann-Neumann, Grundriß der Bakteriologie, V. Aufl.

66. Leschke, in: Spez. Pathol. u. Therapie innerer Krankheiten (KrausBrugsch) I9I9, II, 2.

67. Levy, B. kl. W. I908, 40-4I.

68. Lewandowsky, Zeitschr. f. k1. Med. I900, 40.

69. Linck, Zeitschr. f. O. I920, 79, S. I65.

69a. - Internat. Zentr. f. O. 1920, I7.

69b. - Arch. f. O. I920, I06.

70. Lewkowicz, W. kl. W. I9r8.

71. Lundberg, Acta otorhinologica, ref. Zentr. f. O., Bd. I8, S. I36.

72. Löwe-Meyer, B. k1. W. IgI5, 39.

73. Manasse, Verh. d. otol. Ges, IgI2.

74. Meier, Zeitschr. f. ärztl. Fortb. 1920, 19.

75a. Mygind, Zeitschr. f. O. 72 .

75. Morgenroth-Tugendreich, B. kl. W. I9I6, 20.

76. Neißer-Pollak, Mitt. a. d. Grenzgebieten d. Med. u. Chir. I904, Bd. XIII.

77. Opitz, Mon. f. Kinderh. I919, I5.

78. Ostrowski, Therapie d. Gegenwart I9I9, I0/I I.

79. Passow-Claus, Operationen am Gehörorgan 1920, Sonderdruck, S. 6I. 
80. Pfeiffer, B. kl. W. I918, 40.

81. Plaut-Rehm-Schottmüller, Leitfaden I9I3.

82. Podmaniczky, Therap. Monatshefte IgI8, 2.

83. Pollak, Mitt. a. d. Grenzgebieten d. Med. u. Chir. I907, Bd. XIII.

84. Preysing, Verh. d. otol. Ges. I9I2.

85. Quincke, B. kl. W. I895, 4I, S. 889.

85a. - Über Lumbalpunktion. Die deutsche Klinik 1906, Bd. VI, I.

86. Reichmann, D. Zeitschr. f. Nervenh., Bd. 42.

87. Rolly, D. m. W. I9II, I7.

88. Rosenow, D. m. W. I920, I.

89. Rosenstein, Zentr. f. Chir. IgI9, 22.

90. Schmiegelow, Inter. Zentr. f. O., Bd. XV, S. I42.

91. Schottmüller, Therapie d. Gegenwart i9I7.

92. Schulze, Arch. f. O., Bd. 57/58.

93. Soyka, Arch. f. O., Bd. 108.

94. Spie B, D. m. W. I920, 8.

95. Streit, Arch. f. O. Ior.

95 a. - Arch. f. O., Bd. 83, 89.

95b. - Zur Frage der Ausheilungsmöglichkeit... Urbantschitsch - Festschrift 1920 .

96. Strümpe11, Lehrbuch d. spez. Pathol. u. Ther. I9I2, I8. A., Bd. II, S. 8. 97. Stintzing, im Handb. d. ges. Therapie (Penzoldt-Stintzing), 4. A. IO, Bd. 4, S. I73.

98. Syk, Internat. Zentr. f. O., Bd. XV, S. I73.

99. Tedesco, Großmann, Henke, zit. nach Preysing.

I00. Tuffier, L'analgésie chir. parvoie rhachidienne. Paris 1900.

Ior. Uffenorde, Verh. d. otol. Ges. I9I2, S. 70 u. 74.

Iora. - D. Zeitschr. f. Chir., Bd. II7.

Iorb. - in Katz-Blumenfeld, chirug. Erk. des inneren Ohres, Bd: II,

102. Valentin, Zur Chemotherapie der Pneumokokken-Meningitis. Inaug.-

Diss., Berlin I918.

IO3. Vorschütz, M. m. W. I907, II.

I04. VoB, Verh. d. otol. Ges. IgII, S. 40I. Disk,-Bem.

I04a. - Passows Beitr. IgIo, III, S. 385.

ro4b. - Charitée-Annalen 29.

I04c. - Handb. der Erfahrungen des Weltkrieges (v. Schjerning).

105. Weill-Kafka, W. kl. W. IgII, Io.

I05a. - Med. K1. I9I I, 34.

I06. Westenhöffer-Mühsam, D. m. W. rgr6, 5 I.

I07. Wolff-Lehmann, Jahrb. d. Kinderheilk., Bd. 80, 2.

I08. Zaloziecki, D. Zeitschr. f. Nervenheilkunde, Bd. 46.

Io9. Ziegler, D. Zeitschr. f, Chir., Bd. 65.

IIO. Zimmermann, Z. f. O., Bd. 69.

I Ioa. - Arch. f. O,, Bd. I08. 